\title{
Le complexe sédimentaire de la Galerie des Verviétois (Grotte de Han-sur-Lesse, Belgique)
}

\author{
YvES QUINIF
}

Service de Géologie Fondamentale et appliquée, Faculté Polytechnique, Université de Mons, Belgium; bouqui@skynet.be.

\begin{abstract}
Sedimentary complex in the gallery of "Verviétois" (Cave of Han-sur-Lesse, Belgium). The gallery of "Verviétois" in the cave of Han-sur-Lesse contains a sedimentary series constituted by a lower detrital formation capped by a stalagmitic formation which is characterized by a big flowstone terminated by stalagmites. This series can be followed from the "trou du Salpêtre" entrance to the "the Styx" room where the underground Lesse river flows. The filling is located around 10 metres above the normal water table. This filling is a witness of the hydrodynamic functioning during climatic phases older than the last glaciation. We present here the results of the lithostratigraphic and granulometric study of the detrital formation. It is constituted by two sequences whose particle size decreases from bottom to top. This is a drying sequence terminated by the growing of the stalagmitic formation. By uranium disequilibrium series dating, the flowstone is dated from the isotopic stage 5 for the greater part. The summit has recorded the isotopic stages 3 and 1 . Some lower levels of the flowstone date from the isotopic stages 7 and 9. This indicates that a flow has crossed through the gallery during various climatic cycles.
\end{abstract}

KEYWORDS: Endokarst, detrital filling, granulometry, speleothems, U/Th dating.

RÉSUMÉ. La galerie des Verviétois de la grotte de Han-sur-Lesse renferme une série sédimentaire constituée d'une formation détritique inférieure coiffée par une formation stalagmitique terminée par des stalagmites. Cette série se retrouve depuis l'entrée du « trou du salpêtre » jusqu'à la salle du Styx où coule la Lesse souterraine. Située à une altitude plus haute d'une dizaine de mètres de la surface piézométrique d'étiage, cette série témoigne du fonctionnement de la galerie lors de phases climatiques antérieures à la dernière glaciation. Nous présentons les résultats de l'étude lithostratigraphique et granulométrique de la formation détritique, montrant qu'elle est constituée de deux séquences granodécroissantes faisant partie d'une séquence d'assèchement terminée par la croissance de la formation stalagmitique. Les datations du plancher stalagmitique situent la plus grande partie de ce dernier dans le stade isotopique 5 . Le sommet a enregistré une partie du stade 3 situé au milieu de la dernière glaciation. Le plancher au site de « la forêt » a fourni deux carottages dont la base a fourni des âges appartenant aux stades isotopiques 7 et 9, montrant ainsi que la galerie a connu un fonctionnement durant plusieurs cycles climatiques.

MOTS-CLÉS : Endokarst, remplissage détritique, granulométrie, spéléothème, datations U/Th.

\section{Introduction}

Les dépôts souterrains qui colmatent les conduits karstiques sont depuis plusieurs décennies étudiés pour leur richesse en données paléoenvironnementales, notamment paléoclimatiques (Dandurand et al., 2011 ; Ek \& Quinif, 1988; Lauritzen, 1996 ; Losson et al., 2006 ; Maire \& Pomel, 1997 ; Quinif, 1994 ; 2006 ; Quinif \& Bastin, 1989 ; Quinif et al., 1979 ; Quinif et al., 1995; Quinif \& Maire, 1998). Leur intérêt consiste également à expliciter certaines phases évolutives morpho-sédimentaires sur l'évolution du système karstique, lequel en corollaire fournit des jalons qui doivent être pris en compte lors de l'étude géologique régionale. La galerie des Verviétois dans la grotte de Han-sur-Lesse s'allonge sur plusieurs centaines de mètres depuis la salle Vigneron jusqu'à la salle des Mystérieuses à un niveau globalement supérieur par rapport à la surface piézométrique (Quinif, 1988 ; 1991 ; Quinif \& Bastin, 1986). L'étude de ses dépôts complète ainsi celle du remplissage de la galerie des petites fontaines proche du niveau de base en apportant des données sur des phases climatiques antérieures à l'Holocène et au Pléistocène supérieur (Blockmans et al., 1999).

\section{Le cadre karstique}

\subsection{Le cadre karstique belge}

Les systèmes karstiques belges tels que celui de Han-surLesse résultent de deux phases génétiques. Une première est une fantômisation lors d'une continentalisation sous

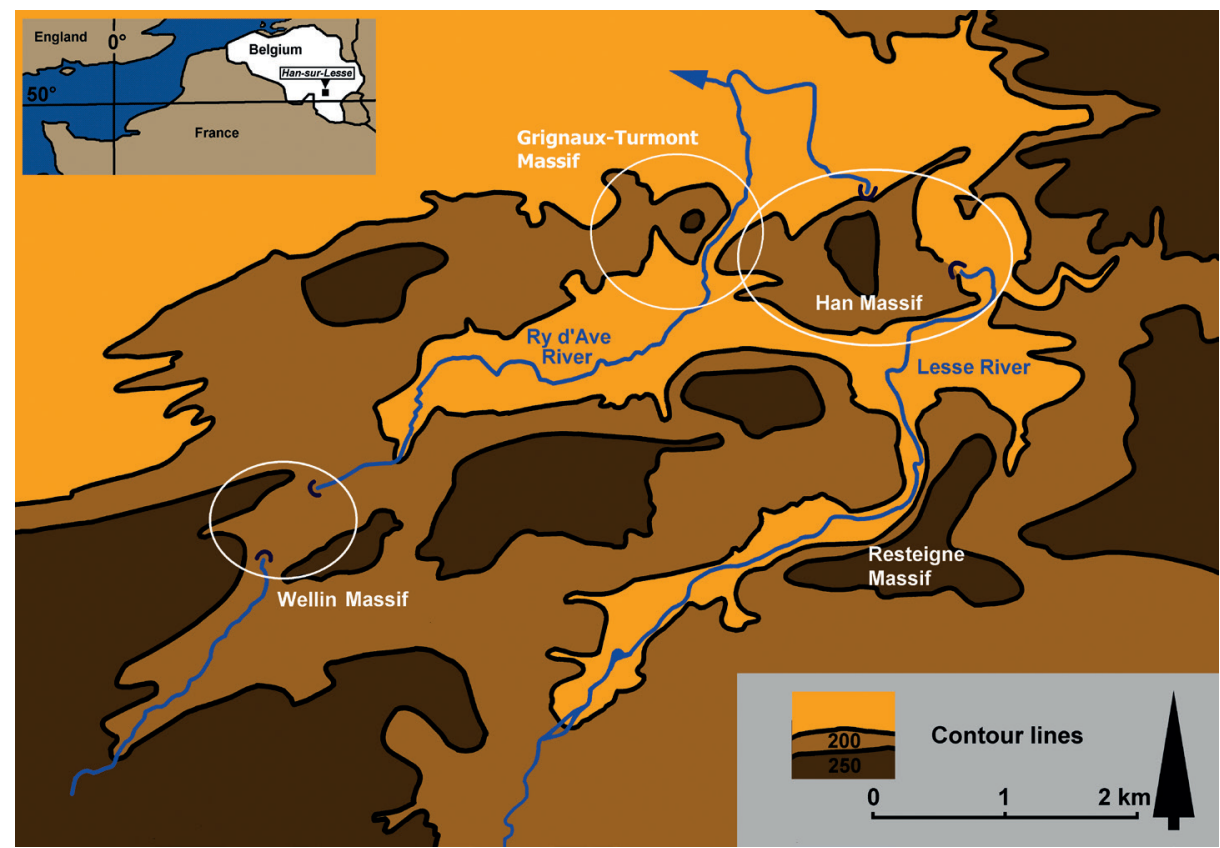

Figure 1. Schéma oro-hydrographique de la région de Han-sur-Lesse - Wellin. La division orographique est basée sur les courbes de niveau de $250 \mathrm{~m}$ et $200 \mathrm{~m}$ pour souligner les principaux reliefs. Géologiquement, les courbes de niveau fermées délimitent les 4 massifs calcaires et les 4 traversées épigénétiques avec 2 systèmes karstiques de type recoupement souterrain de méandre : celui du Ry d'Ave dans le massif de Wellin et celui de la Lesse dans le massif d'Han.

Figure 1. Oro-hydrographic sketch of the area of Han-sur-Lesse - Wellin. The orographic division is based on the contour lines of 250 and $200 \mathrm{~m}$ to emphasize the principal reliefs. From a geological point of view, the closed contour lines draw the four limestone massifs with four river crossings and two karstic systems: the Ry d'Ave River in the Wellin Massif and the Lesse River in the Han Massif which are meander crosscheckings. 


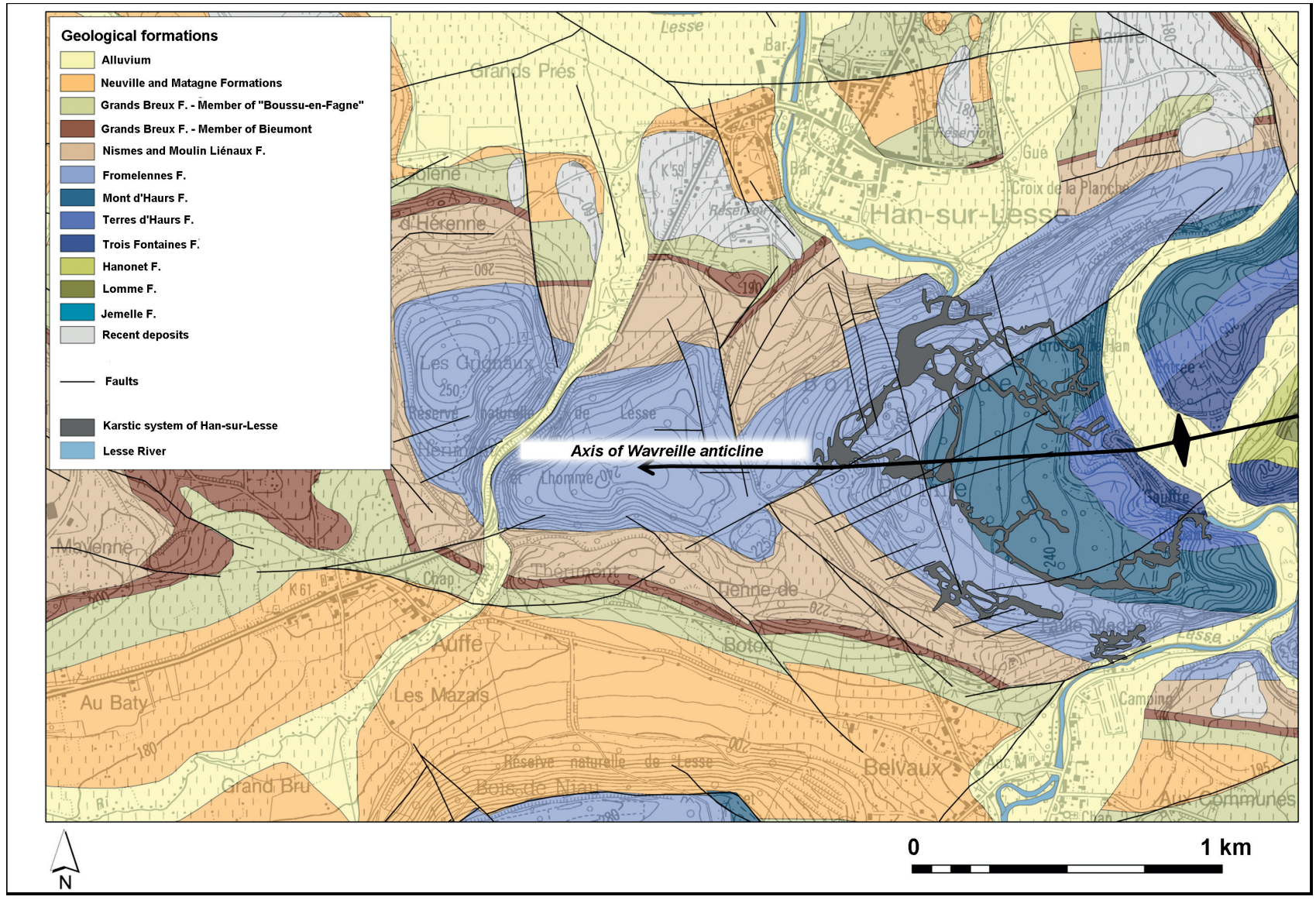

Figure 2. Carte géologique des massifs de Han et des Grignaux-Turmont. Cette carte est inspirée de la nouvelle carte géologique de Wallonie (Bonniver, 2011, d'après Blockmans \& Demoulin, sous presse). Les formations calcaires givétiennes sont en bleu. Formations de Jemelle, de la Lomme et de Hanonet : Eifelien ; Formations de Trois Fontaines, des Terres d'Haurs, du Mont d'Haurs, de Fromelennes : Givétien ; Formation de Nismes, de Moulin Liénaux, des Grands Breux, de Neuville et de Matagne : Frasnien.

Figure 2. Geological map of the massifs of Boine and Grignaux-Turmont. This map is inspired from the new geological map of Wallonia (Bonniver, 2011, from Blockmans \& Demoulin, in press). The Givetian limestone formations are in blue. Jemelle, Lomme and Hanonet Formations : Eifelian ; Trois Fontaines, Terres d'Haurs, Mont d'Haurs and Fromelennes Formations : Givetian ; Nismes, Moulin Liénaux, Grands Breux, Neuville and Matagne Formations : Frasnian.

climat tropical daté du Mésozoïque (Spicer, 2003 ; Yans, 2003). La seconde suit la surrection néogène de l'Ardenne avec encaissement du réseau hydrographique, amenant la vidange partielle des fantômes de roche par l'installation d'un potentiel hydrodynamique. Il en résulte deux types morphohydrogéologiques de systèmes : des recoupements souterrains de méandres comme celui de Han-sur-Lesse et des systèmes " chantoirs - résurgences » dans lesquels des ruisseaux de plateaux se perdent dans le calcaire pour résurger au creux des vallées épigénétiques. Ce type de karst peut donc être défini comme un karst sur relief appalachien (Derruau, 1974).

\subsection{Le système de la Lesse souterraine à Han-sur-Lesse}

En rencontrant les barres calcaires givétiennes en relief au sein de pélites eifeliennes et frasniennes, au sortir du massif ardennais, la Lesse franchit le premier relief calcaire à Resteigne par une cluse aérienne, traverse le synclinal schisteux de Belvaux et butte contre l'anticlinal calcaire de Boine qu'elle recoupe souterrainement par le système karstique de Han-surLesse. D'autres traversées épigénétiques de barres calcaires sont présentes dans la région, comme le Ry d'Ave à Wellin (Fig. 1 ; Lannoote et al., 2013).

La région de Han-sur-Lesse appartient au socle paléozoïque de l'Ardenne s.l. tectonisé par l'orogenèse hercynienne (Blockmans \& Demoulin, sous presse ; Boulvain et al., 2009 ; Boulvain \& Pingot, 2011 ; Delvaux de Fenffe, 1985 ; 1989 ; 1998). Les formations présentes regroupent les calcaires du Givétien qui constituent l'ossature karstifiée des paysages, et les pélites de l'Eifelien et du Frasnien. La structure se compose essentiellement de plis orientés est-ouest, hachés par endroits de failles (Fig. 2) et d'un grand nombre de joints (diaclases et joints tectoniques s.s. essentiellement en $\mathrm{N} 60^{\circ} \mathrm{E}$ et $\mathrm{N} 150^{\circ} \mathrm{E}$ ). Ce socle a été aplani durant la première partie de l'ère mésozoïque pour présenter une morphologie monotone au Crétacé. Sur les zones émergées, le climat tropical favorisait le développement d'une forêt avec une très forte érosion chimique dont les kaolins de Transinne en sont une illustration sur socle quartzo-phylladeux.

Le système karstique de Han-sur-Lesse est donc un recoupement souterrain de méandre. Il comprend plusieurs passages spéléologiques au travers du massif, soit en suivant la rivière souterraine permanente moyennant le franchissement de siphons, soit par des galeries sèches qui s'étendent sur deux niveaux altitudinaux (Quinif, 2015 ; Quinif \& Vandycke, 2001 ; Van Den Broeck et al., 1910) (Fig. 3). Le niveau inférieur débute au Trou d'Enfaulne et rejoint la rivière dans la salle d'armes. Il reste à quelques mètres au-dessus de la surface piézométrique d'étiage et connaît ainsi un ennoiement lors de crues. La galerie des Verviétois fait partie du niveau supérieur de la grotte de Han qui débute par l'entrée du trou du salpêtre, continue par une suite de galeries et de salles (des scarabées, du renard, de la grenouille) jusqu'à la salle Vigneron (Fig. 4). La galerie des Verviétois débute à cet endroit. C'est une succession de parties horizontales et de dépressions liées à des entonnoirs de subsidence. Deux élargissements qui méritent le nom de salles interrompent ce cheminement. Le premier est le chaos, cône d'éboulis atterrissant sur la galerie qui présente à cet endroit un entonnoir de subsidence. Le second comprend la stalagmite appelée le minaret. Cet élargissement est axé sur deux grands joints verticaux $\mathrm{N} 150^{\circ} \mathrm{E}$ se développant 


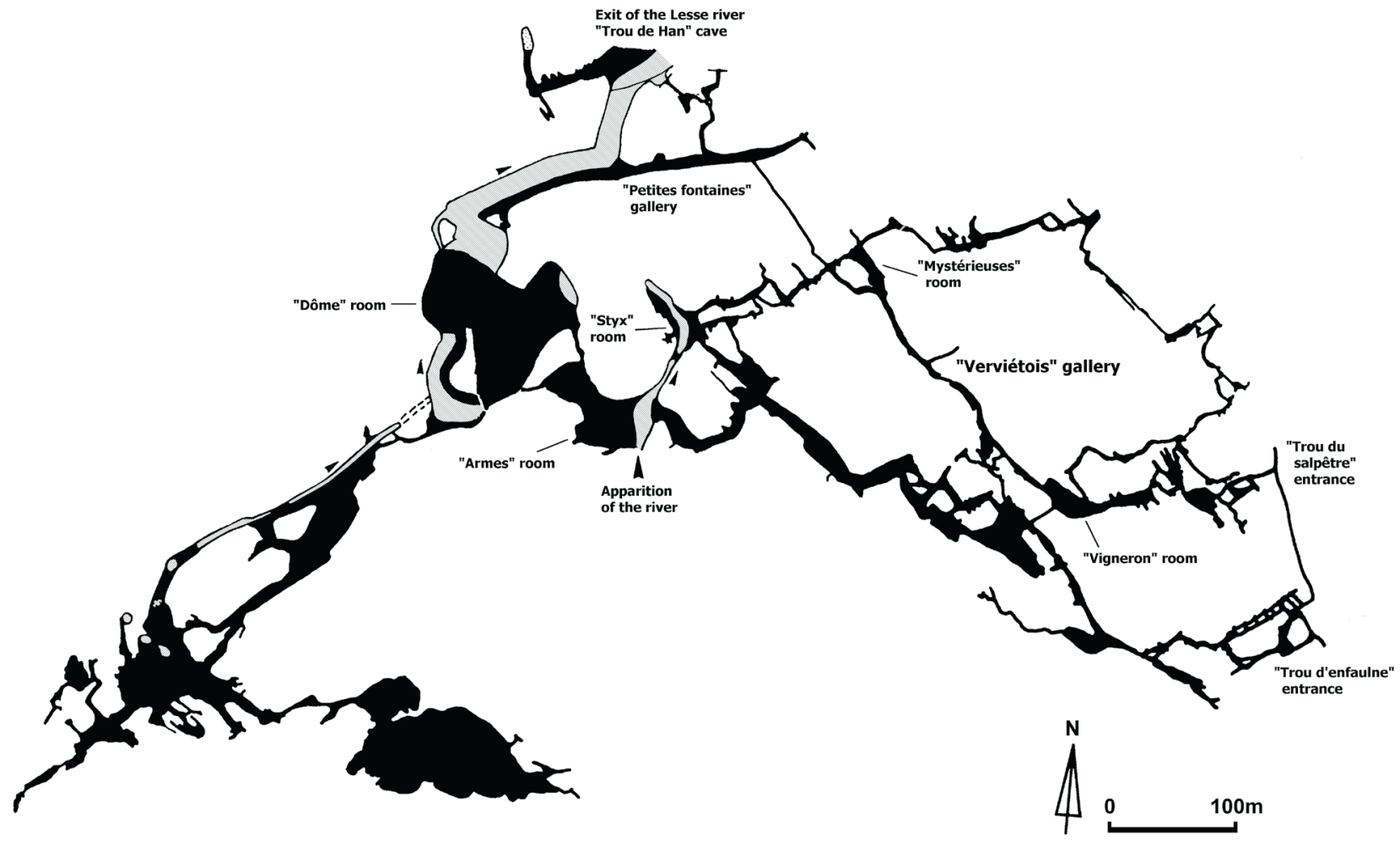

Y. Quinif, 1987

Figure 3. Plan général de la grotte de Han-sur-Lesse avec la localisation de la galerie des Verviétois et des autres sites mentionnés dans le texte. Figure 3. General map of the cave of Han-sur-Lesse with the location of the "Verviétois" gallery and other sites indicated in the text.

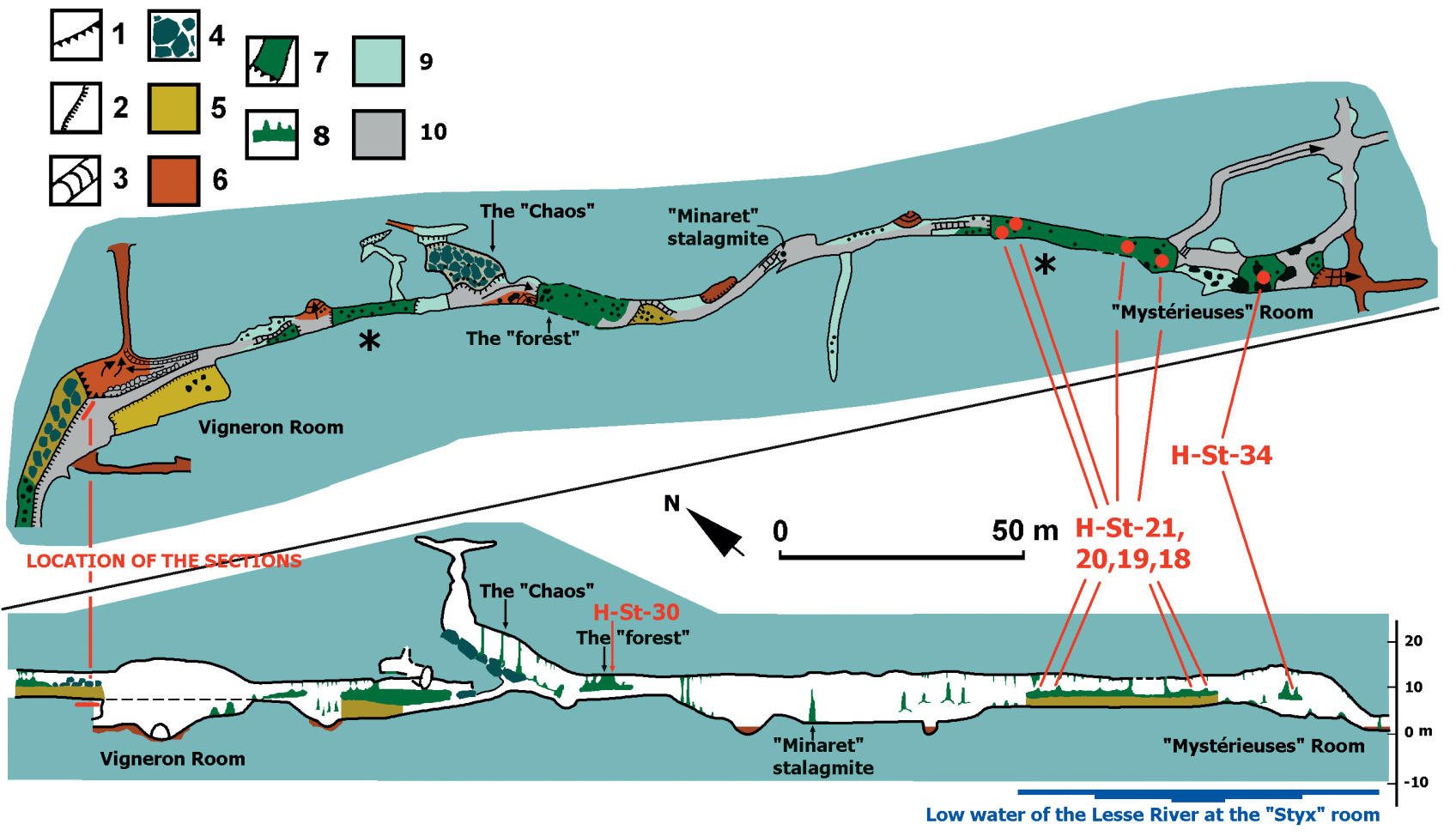

Figure 4. Plan et coupe longitudinale de la galerie des Verviétois. 1. Escarpement de plus de $5 \mathrm{~m} ; 2$. Escarpement inférieur à $5 \mathrm{~m} ; 3$. Pente du sol ; 4. Eboulis ; 5. Sédiments détritiques ; 6. Limons des crues actuelles ; 7. Planchers stalagmitiques en plan; 8. Planchers stalagmitiques et stalagmites en coupe ; 9. Roche mère ; 10. Parcours touristique. Les grands planchers stalagmitiques sont à la même altitude et coiffent une formation détritique. Les parties inférieures de la galerie résultent d'une subsidence entre les deux niveaux de galeries comme la salle Vigneron et les différents entonnoirs de subsidence qui accident la galerie. Les deux astérisques indiquent les endroits où un courant plus rapide a engendré des chenaux.

Figure 4. Map and longitudinal section of the "Verviétois" Gallery. 1. Escarpment higher than $5 \mathrm{~m} ; 2$. Escarpment lower than $5 \mathrm{~m}$; 3 . Slope of the ground; 4. Fallen blocks; 5 . Detrital sediments; 6 . Loam due to the recent high floods; 7. Flowstones; 8 . Flowstones and stalagmites in section; 9. Bedrock; 10. Touristic path. The sections of the great flowstones are at the same altitude and cap a detrital formation. The lower parts of the gallery are collapses between two levels of galleries like the "Vigneron" room or decanting to lower voids. The two asterisks indicate where faster current generated channels. 


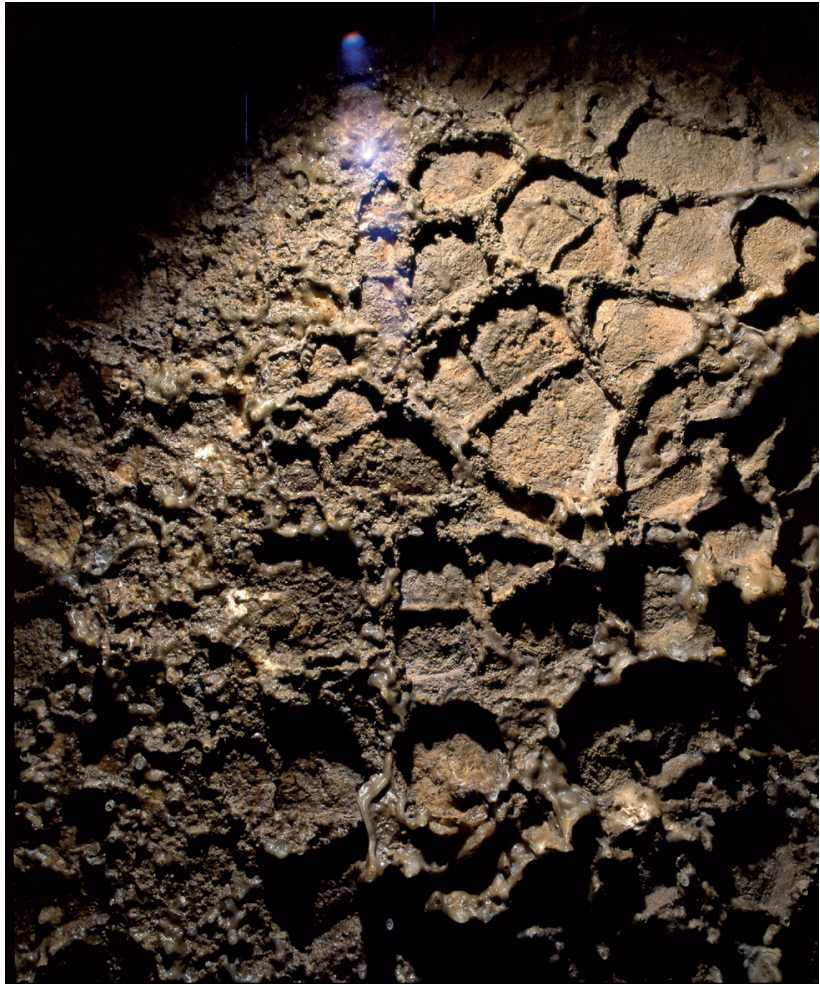

Figure 5. Surface inférieure du plancher stalagmitique, dans la section en amont de la salle des Mystérieuses, montrant les empreintes des fentes de dessiccation du sommet du remplissage détritique. La dimension moyenne des polygones est de $15 \mathrm{~cm}$. Photo de Guy Deflandre.

Figure 5. Lower surface of the flowstone near the "Mystérieuses" room. Marks of mudcracks are visible at the top of the detrital filling. The average size of the polygons is $15 \mathrm{~cm}$. Photo by Guy Deflandre. parallèlement à quelques mètres de distance. La salle des Mystérieuses constitue la terminaison de la galerie.

\subsection{La Galerie des Verviétois}

Hormis le fait de son altitude, la galerie présente une unité au travers du complexe sédimentaire qu'elle renferme. $\mathrm{Ce}$ complexe comprend une formation détritique surmontée d'une formation stalagmitique formée d'un plancher stalagmitique de très grande ampleur supportant des spéléothèmes divers : stalagmites, disques, coulées. Ce remplissage constitue le cœur de cette étude. Les données concernant la formation détritique sont nouvelles, les données sur la formation stalagmitique sont reprises d'études antérieures (Quinif, 1991 ; 1996 ; 2006). Les formes pariétales sont surtout constituées de coupoles en parois ou à la voûte au détriment des joints $\mathrm{N} 150^{\circ} \mathrm{E}$. Certaines portions de parois montrent des formes en cuillère assimilables à des coups de gouge altérés. La galerie étant sécante à la direction de la stratification, elle recoupe une grande partie de la Formation du Mont d'Haurs (Fig. 2). Des indices de fantômisation, comme des strates altérées, sont bien visibles et sont confirmés dans d'autres parties de la galerie jusqu'à la salle du trophée (Quinif, 2015).

\section{La formation détritique}

\subsection{Vue d'ensemble}

La salle Vigneron résulte de l'effondrement d'un plancher rocheux entre la galerie des Verviétois et une galerie inférieure. Cette disposition permet d'étudier la formation détritique depuis la base jusqu'au sommet, alors que la base est généralement masquée. Dans la plus grande partie de la salle, la formation détritique est recouverte par des blocs éboulés, caractéristiques d'une salle, tandis que dans la galerie, elle est coiffée de la formation stalagmitique.

Le passage touristique qui précède la salle des Mystérieuses est le plus typique : il a été creusé au travers des sédiments détritiques sous le plancher stalagmitique qui est ainsi visible
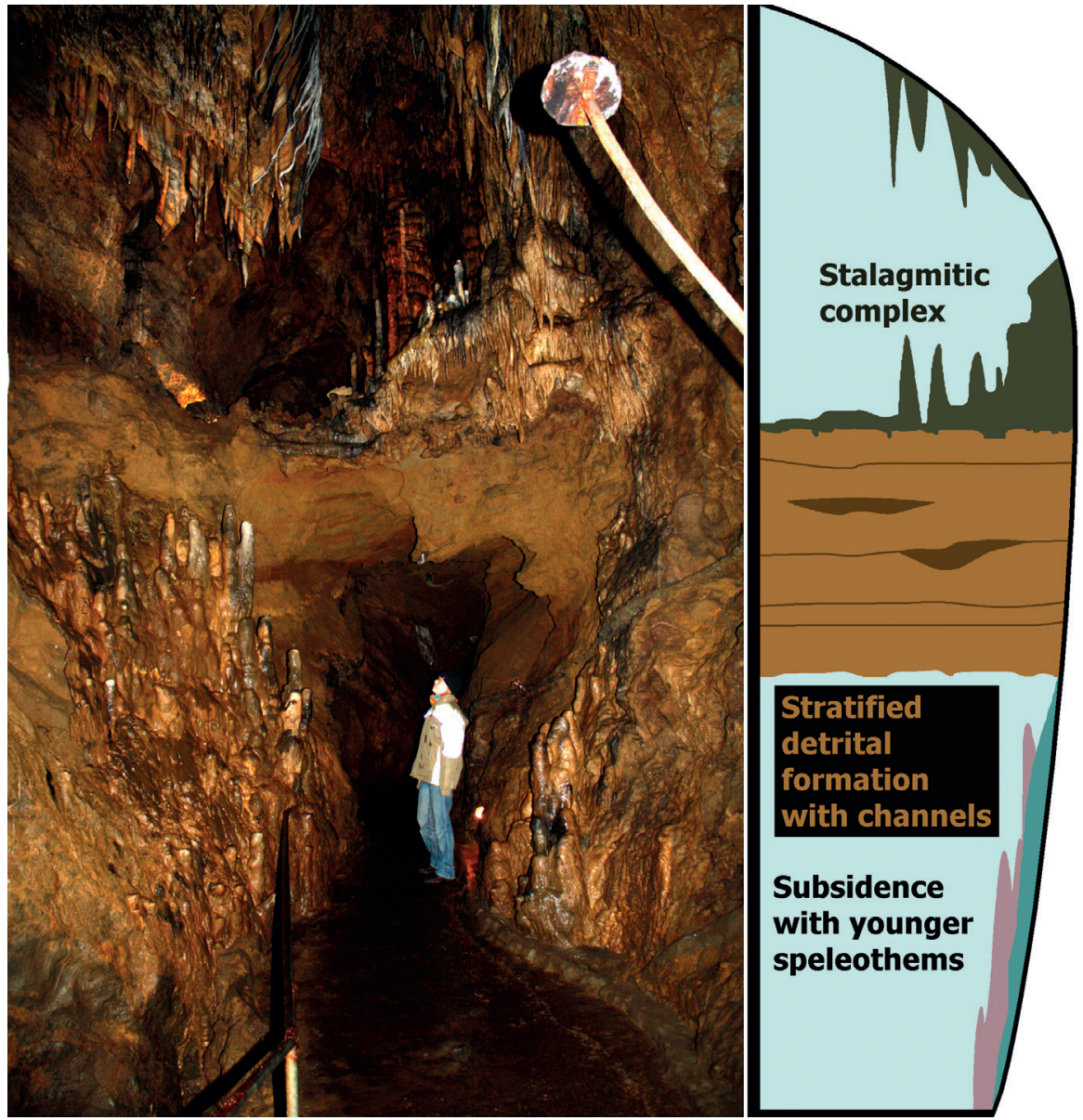

Figure 6. Coupe du remplissage dans la galerie des Verviétois. La photo est prise dans un bas niveau dû à la subsidence dans des vides inférieurs. Ce mouvement peut être daté par les stalagmites qui croissent sur les flancs de l'affaissement. Le plancher qui constitue la base de la formation stalagmitique date du stade isotopique 5 .

Figure 6. Sedimentary section of the filling in the "Verviétois" gallery. The view is taken in a low level provoked by a subsidence into a lower void. This movement can be dated due to the speleothems which are growing on the flank of the collapse. The flowstone which constitutes the basis of the stalagmitic formation dates from the isotopic stage 5 . 


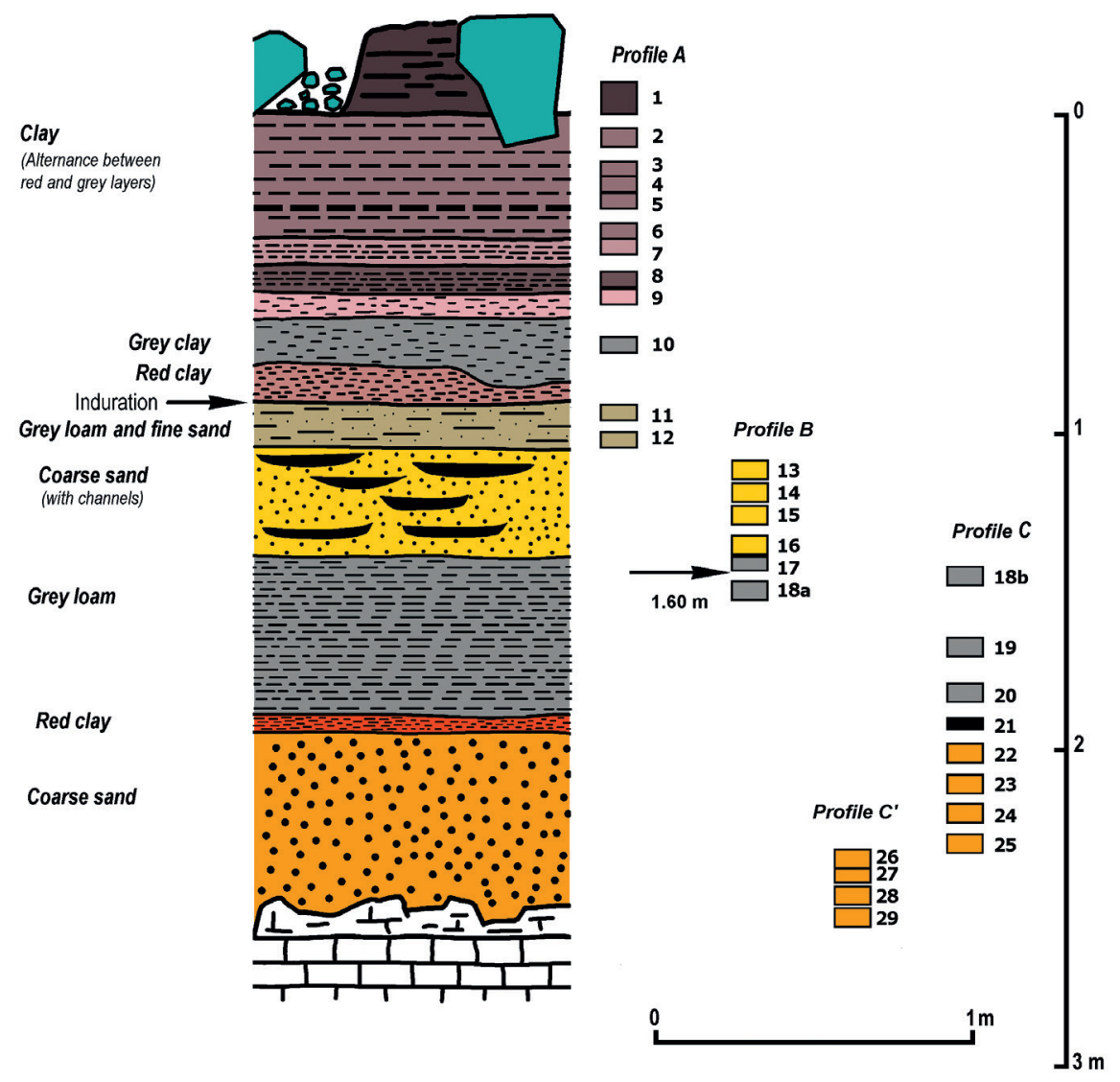

Figure 7. Coupe de la salle Vigneron. Les couleurs sont purement figuratives. Cette coupe théorique résulte de la combinaison de trois profils réalisés en raison des difficultés techniques d'échantillonnage (face au vide).

Figure 7. Sedimentary section of the "Vigneron" room. The colours are purely indicative. This theoretical section results from the combination of three profiles created due to the difficulties of sampling (facing the vertical wall).

$\mathrm{du}$ dessous et montre l'empreinte de fentes de dessiccation, trace du sommet du remplissage sur lequel il s'est développé (Fig. 5). Il constitue notre coupe holotype de la galerie (Fig. 6). De plus, le plancher a été carotté à plusieurs endroits, des carottes ayant fait l'objet de datations (Quinif, 1991 ; 1996).

\subsection{La coupe de la salle Vigneron}

\subsubsection{Lithostratigraphie}

Cette série est composée de sédiments détritiques allant de sables fins à moyens jusqu'à des silts (Fig. 7). Deux séquences peuvent y être observées. La séquence inférieure est la plus

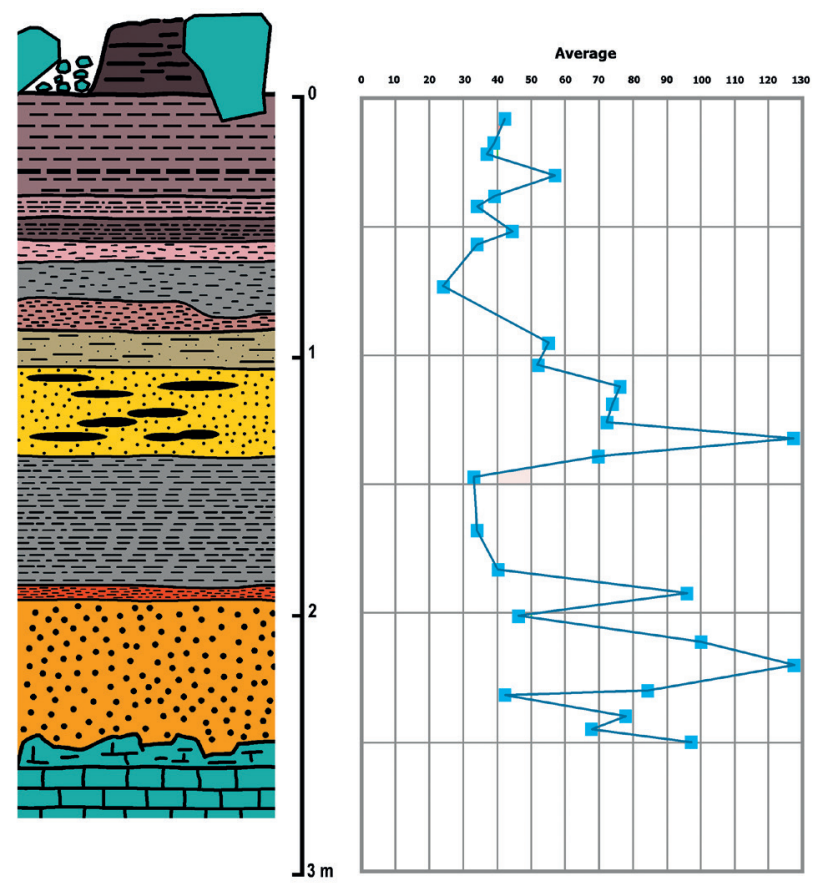

Figure 8. Evolution stratigraphique de la moyenne. Figure 8. Stratigraphic evolution of the mean value. grossière avec une succession de sables se terminant par des limons. La seconde séquence débute également par des sables présentant des chenaux centimétriques et se termine par des sédiments fins. D'une manière générale, ces deux séquences sont à granulométrie décroissante et témoignent d'une diminution de la capacité de transport. Cette caractéristique est bien marquée sur un log de la moyenne arithmétique (Fig. 8)

\subsubsection{Etude granulométrique}

\section{a. Méthodologie}

Les échantillons sont séchés et décarbonatés, une fraction de 100 grammes est prélevée, placée dans un bain de dispersant (hexamétaphosphate de sodium) et lavée sur une colonne comprenant les tamis de 20-25-32-40-50-63 et $80 \mu \mathrm{m}$. Le refus à $80 \mu \mathrm{m}$ est séché et tamisé à sec sur une colonne comprenant les tamis de 100-125-160-180-200-250-310-400-500 $\mu \mathrm{m}$. Chaque fraction est pesée et gardée pour l'examen minéralogique et morphologique. Les poids des fractions inférieures à $20 \mu \mathrm{m}$ sont estimés par extrapolation mathématique au moyen d'un polynôme du troisième degré testé sur des échantillons témoins par la méthode par décantation. Cela permet de calculer la médiane avec une bonne précision, largement utilisée pour l'interprétation de Passega.

Cette méthode synthétique (Passega, 1957 ; 1964 ; 1977 ; Passega \& Byramjee, 1969 ; Rivière, 1977) permet une bonne interprétation de l'hydrodynamisme grâce à un diagramme bilogarithmique entre le premier centile (1C) et la médiane $(\mathrm{Me})$. Le premier centile représente la dimension des grains de telle façon que $1 \%$ de l'échantillon soit plus grand que cette taille, ce paramètre étant une approximation de la taille maximale des grains transportés par le courant avant leur dépôt. La médiane (Me), qui représente la dimension des grains telle que $50 \%$ de l'échantillon soit plus grand et plus petit que cette taille, caractérise la distribution globale des grains car elle divise la distribution en deux parties de même masse. Elle est fonction de la turbulence du courant durant le dépôt. Rivière (1977) démontre que cette représentation est significative car une grande partie de l'information se retrouve dans deux paramètres : le premier centile et la moyenne arithmétique. Comme il existe une très forte corrélation 


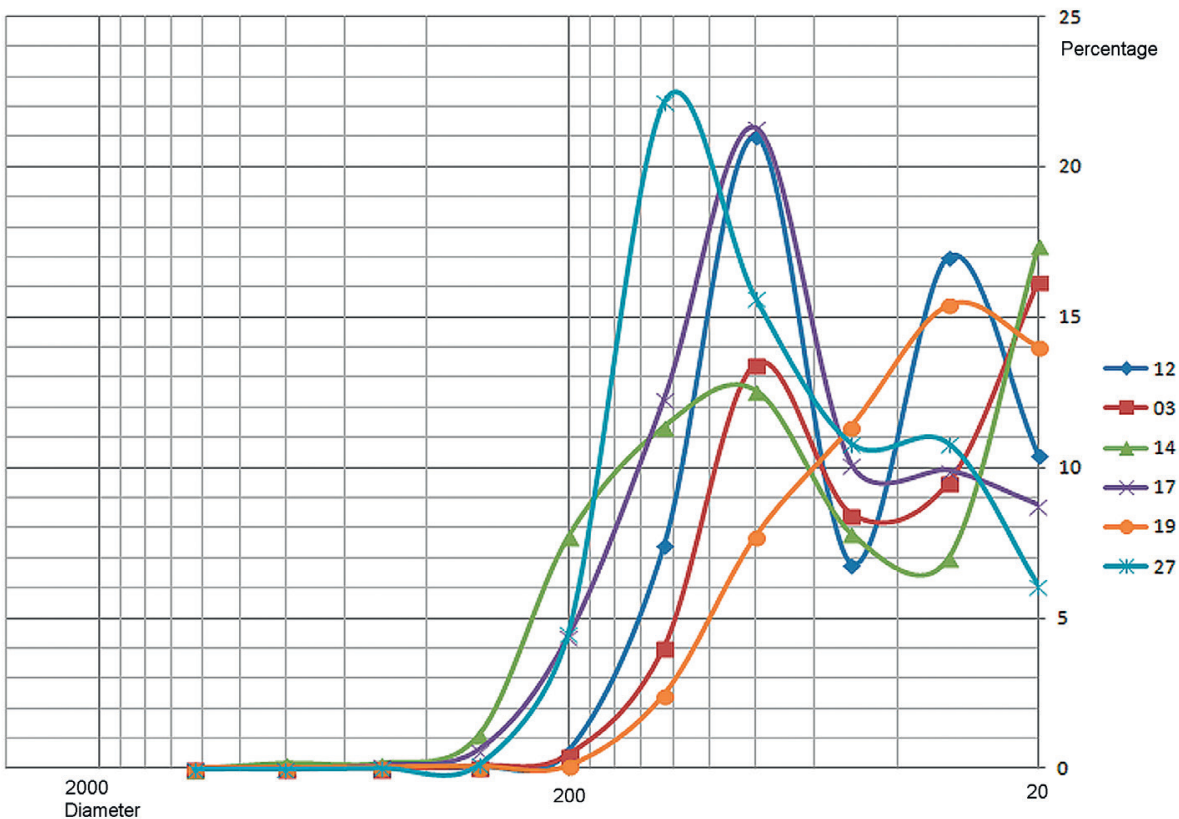

Figure 9. Courbes granulométriques représentatives des faciès de la coupe de la salle Vigneron. 03 est représentatif des argiles supérieures, $12 \mathrm{du}$ limon gris supérieur, $14 \mathrm{du}$ sable médian, 17 et 19 des limons gris inférieurs, $27 \mathrm{du}$ sable grossier inférieur.

Figure 9. Granulometric curves showing the facies in the section of the "Vigneron 》 room. 3 is representative of the upper clays, 12 of the grey top loam, 14 the middle sand, 17 and 19 of the lower grey loams and 27 the lower coarse sand. linéaire entre cette moyenne arithmétique et la médiane, on retrouve ainsi la représentation de Passega.

Plusieurs zones apparaissent dans le diagramme de Passega. La "suspension gradée » représente les courants de fond (branche QR). La dimension des particules transportées et leur concentration diminue de bas en haut. Ce dépôt se met en place quand la charge dépasse la capacité de transport. La « suspension uniforme " se place au dessus de la suspension gradée (branche SR). Dimensions et concentration des particules sont uniformes. Lorsque le courant diminue, ces particules passent dans la suspension gradée. Leur dépôts correspond à des dépôts de type lit majeur, lorsque les conditions de décantation prédominent, entraînant une dispersion de la médiane pour un même domaine des premiers centiles. La branche PO exprime le dépôt de grains de plus en plus grossiers, avec entrainement de la partie fine. La médiane augmente alors que le premier centile reste constant. La branche PQ constitue la transition où on observe un apport de grains grossiers. Enfin, la dernière branche regroupe des grains grossiers dans un contexte d'évacuation des fines et de dépôts d'éléments transportés par roulement. Les plages séparées dans les domaines très fins correspondent à des conditions de décantation totale.
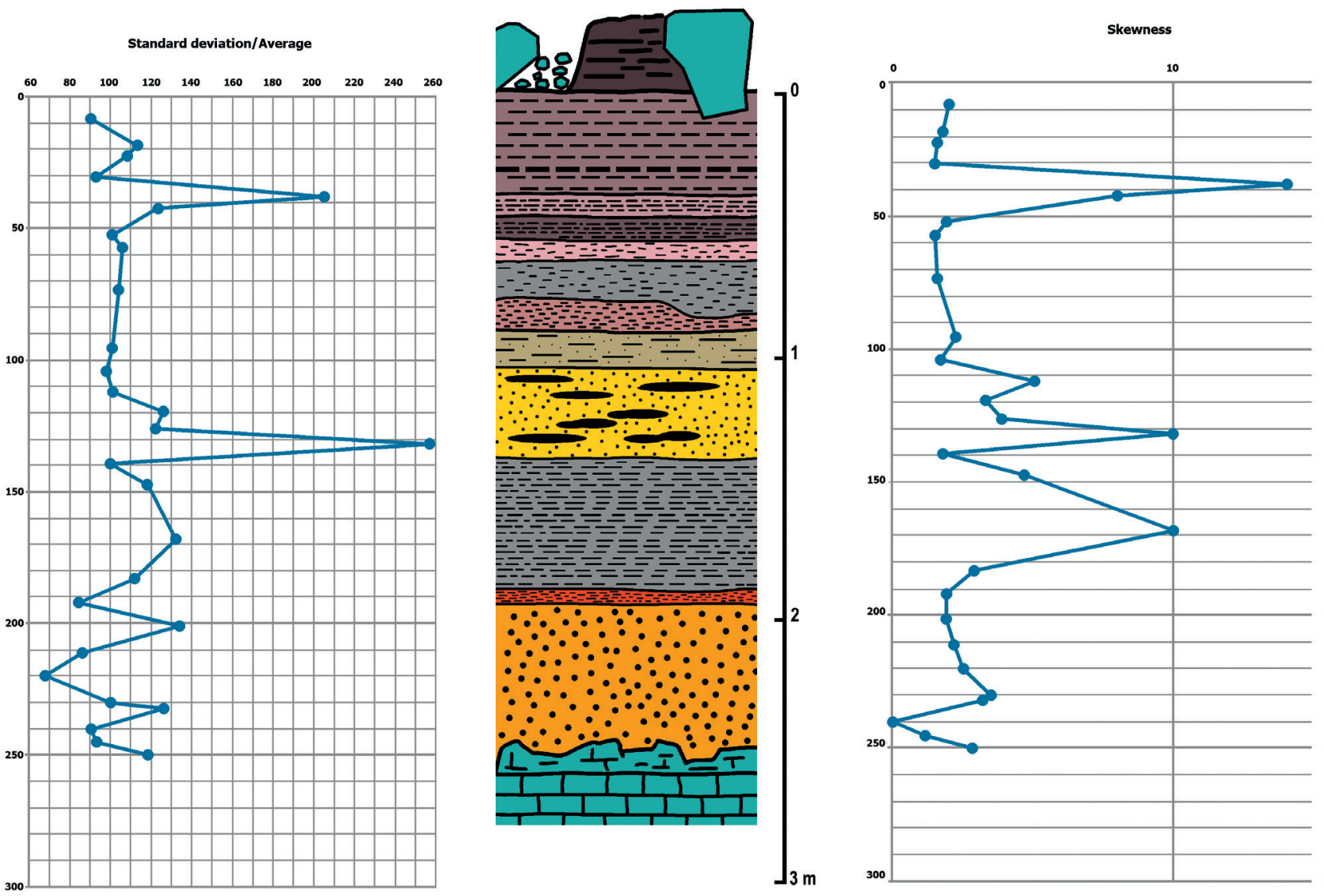

Figure 10. Evolution stratigraphique de l'écart-type normalisé à la moyenne arithmétique à gauche et du facteur de symétrie (skewness) à droite. Figure 10. Stratigraphic evolution of the normalized gap time to the arithmetic mean value to the left and the skewness factor to the right. 

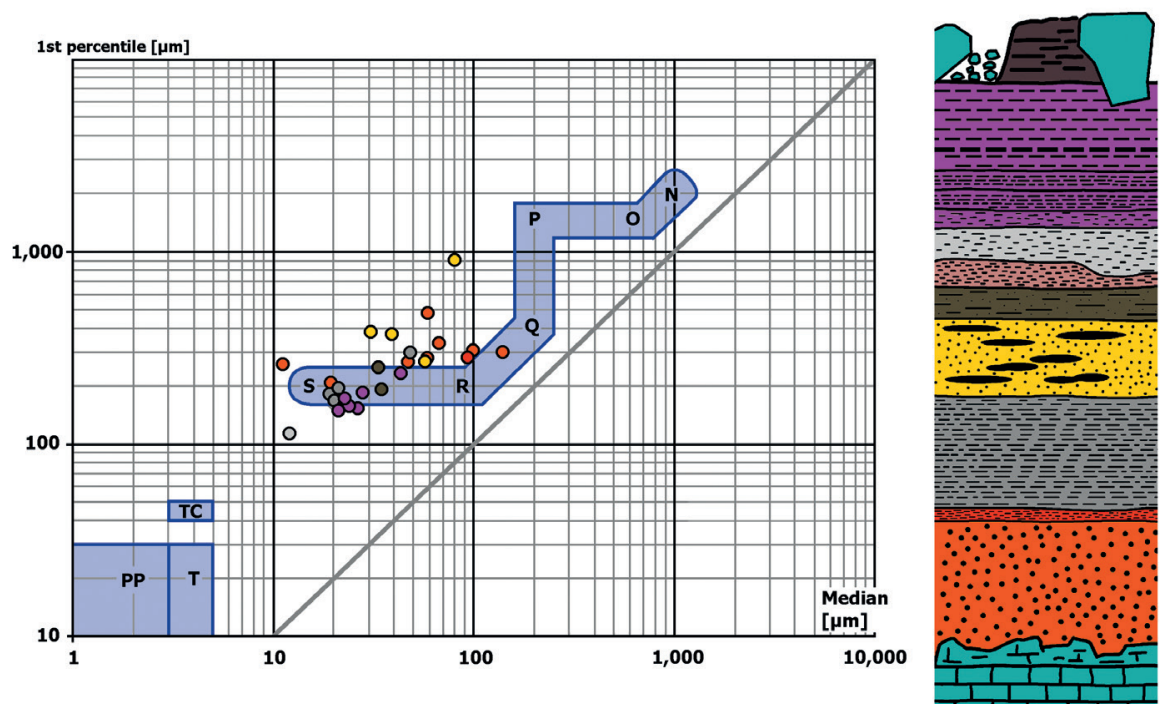

Figure 11. Diagramme de Passega pour les échantillons de la salle Vigneron. Les couleurs des échantillons se rapportent aux couleurs sur la coupe. La signification des différentes zones est explicitée dans le texte.

Figure 11. Passega's graph for the samples of the "Vigneron" room. The colours for the samples on the diagram are linked to the same colour in the lithostratigraphic section. The meaning of the different areas is explained in the text.

\section{b. Courbes granulométriques}

En grottes, les courbes sont rarement unimodales (Fig. 9). Cela tient à la complexité de la géométrie du chenal, vu les contraintes imposées par l'encaissant rocheux. Il faut aussi tenir compte de l'alternance crues - décrues fréquentes en grotte qui associe ainsi plusieurs modes. Aussi, les diverses méthodes d'analyses sont souvent confrontées à la question de choix : faut-il traiter la courbe globale ou les modes séparément ? Nous présenterons ainsi l'un ou l'autre résultat en les comparant et en les critiquant dans l'interprétation de Passega.

c. Evolution stratigraphique des paramètres granulométriques La moyenne est une expression de l'énergie de transport. Son évolution stratigraphique (Fig. 8) explicite les deux cycles sédimentaires. L'échantillon le plus grossier des sables médians a été extrait d'un chenal. Les sables inférieurs sont plus grossiers d'une manière générale. La courbe de l'écarttype normalisé à la moyenne arithmétique (Fig. 10) met en évidence deux points qui s'écartent vers des valeurs plus grandes ce qui exprime une plus grande dispersion des grains ; l'un d'eux est le sable de chenal de l'ensemble moyen. Les limons supérieurs sont d'une manière générale assez bien classés : le courant devenait de plus en plus calme et régulier. Les sables inférieurs sont plus variables, montrant une hydrodynamique plus irrégulière.

Le facteur skewness est la symétrie de la courbe. Une valeur nulle correspond à une courbe de gauss totalement symétrique, les valeurs positives à une courbe dissymétrique vers les valeurs plus grossières. En d'autres termes, il y a moins de fractions grossières. Par exemple, le point à skewness le plus élevé est l'échantillon le plus fin. Une constatation intéressante est la plus grande dispersion des points des sables moyens comparés aux points des sables inférieurs. Les sables moyens comprennent des sédiments recreusés au long de leur évolution par des chenaux, ce qui donne une hétérogénéité au niveau du rapport entre les fractions grossières et les fractions fines. Par contre, les sables inférieurs, bien que déposés avec une hydrodynamique assez variable, sont plus homogènes quant à leur granulométrie.

\section{d. Méthode de Passega}

Diagramme complet. Les échantillons de la coupe Vigneron pris dans leur totalité (Fig. 11) et classés comme argiles ou limons à l'affleurement se positionnent autour de la zone SR, dépôts de suspension uniforme témoignant d'une vitesse de fond trop faible pour produire un quelconque classement, comme les dépôts de lit majeur. Les sables supérieurs s'en éloignent quelque peu, avec un échantillon plus proche de la zone PQ, dans laquelle le roulement des grains prend le pas. Les sables inférieurs sont plus dispersés mais se rapprochent de la zone RQ, dépôts de suspension gradée apparaissant lorsque la charge dépasse la capacité de transport. D'une manière générale, l'ensemble de ce dépôt marque une diminution de la capacité de transport, probablement liée à l'élargissement du lit à l'entrée de la salle Vigneron. Le point à l'écart dans les sables supérieurs se rattache à un chenal où la concentration
Figure 12. Diagramme de Passega pour quelques échantillons de la salle Vigneron. Les courbes ont été déconvoluées et chaque mode a été recalculé pour fournir le premier centile et la médiane.

Figure 12. Passega's graph for some samples of the "Vigneron" room. A deconvolution has been made and for each mode, the first centile and the median point have been calculated.

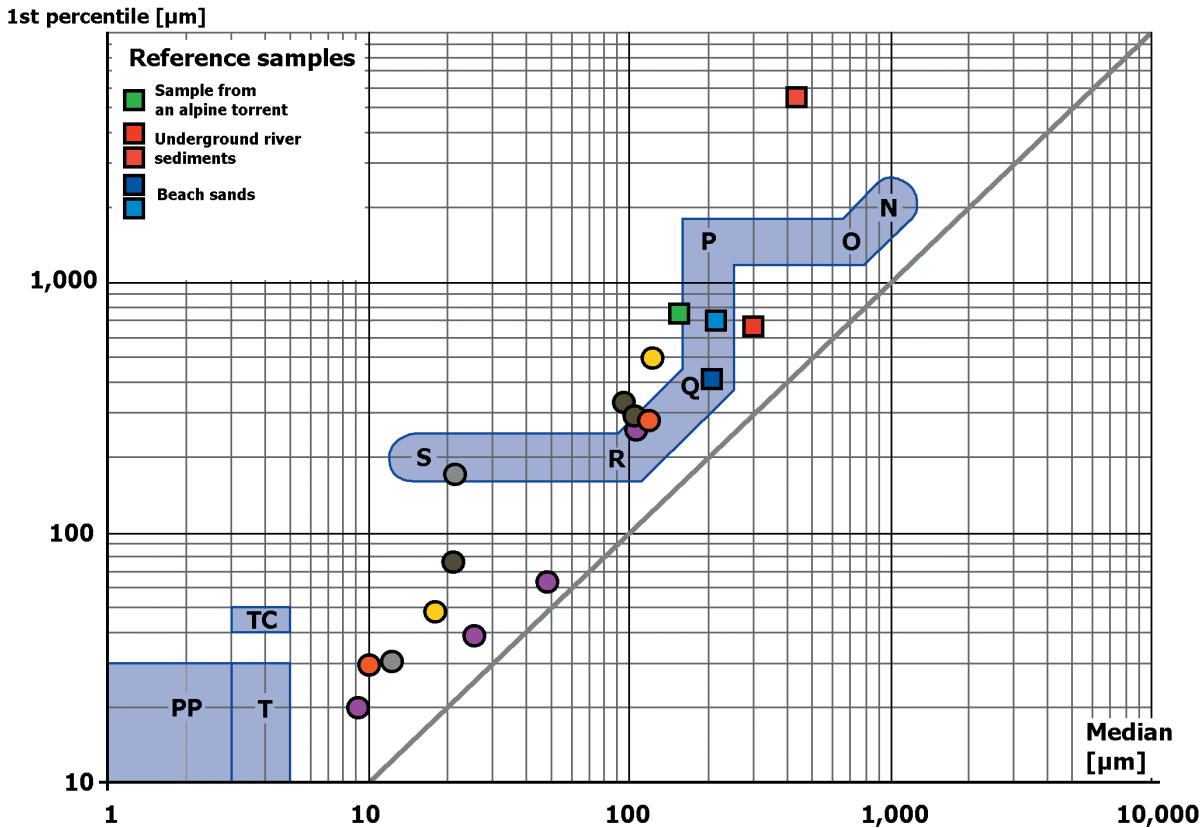




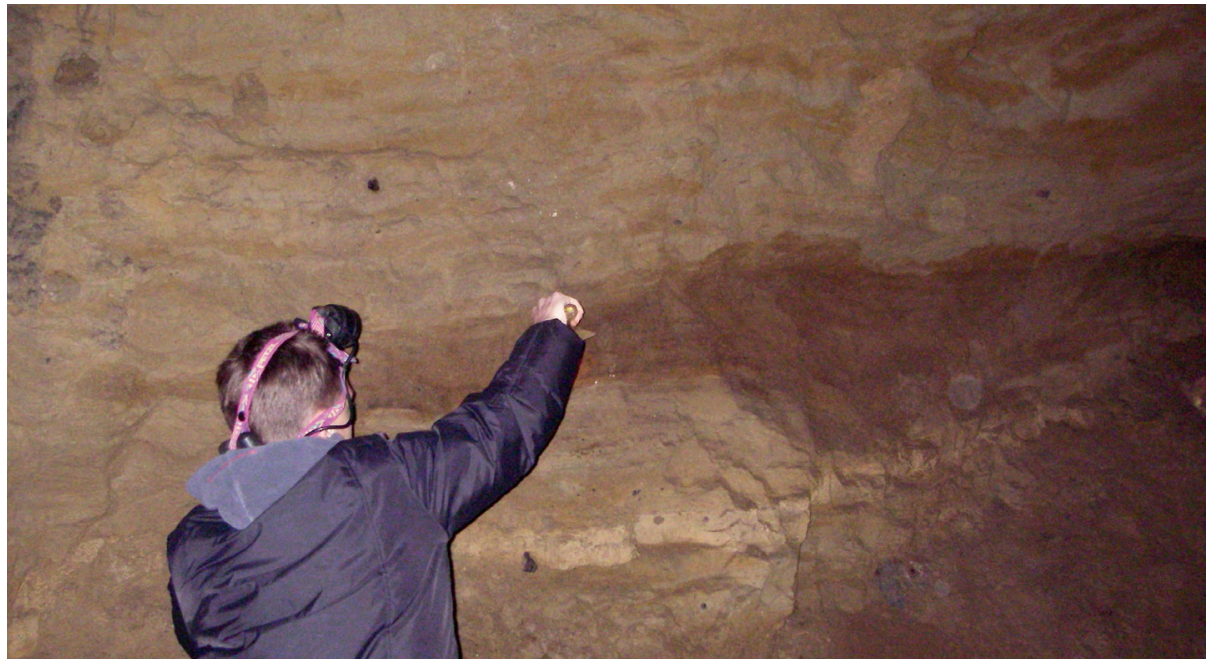

Figure 13. Echantillonnage d'un chenal, situé dans les sables médians, 50 mètres en aval de la coupe de la salle Vigneron. Figure 13. Sampling of a sandy channel, in the medium sands, 50 metres downstream from the "Vigneron" room.

du courant produit un dépôt par roulement. On a l'image d'un courant se divisant en bras multiples recreusant le dépôt précédent.

Déconvolution. Quelques modes obtenus après déconvolution de courbes multimodales d'échantillons ont été représentés sur le diagramme de Passega (Fig. 12). On constate que le mode supérieur se positionne très bien sur la branche RQ de suspension gradée. Un limon reste à l'extrémité de la branche RS de suspension uniforme. Les modes plus fins se dirigent vers les zones de décantation sans les atteindre. Rappelons néanmoins que ces zones ont été définies sur des échantillons marins, comme par exemple «l'argile des grands fonds » dans des contextes très éloignés du nôtre. Des carrés représentant des échantillons test ont été ajoutés afin de comparer nos échantillons d'études avec des échantillons dont les conditions actuelles de sédimentation sont bien connues. Les deux carrés rouges se rapportent à des sédiments fluviatiles actuels (grotte des Échelles, Massif de la Chartreuse, Savoie, France), les deux échantillons bleus sont des sables de plage et l'échantillon vert un sable fluviatile d'une laisse dans un torrent alpin (torrent du Giffre, Samoëns, Hautes Alpes, France). Il y a mélange entre un sable fin déposé lorsque la charge dépasse la capacité de transport et un limon correspondant à une fin de crue.

\subsection{Autres observations sur l'ensemble détritique}

Le chemin touristique a été réalisé par creusement dans l'ensemble détritique afin de préserver l'ensemble stalagmitique supérieur. Cela permet de pouvoir examiner des fragments de coupes à divers endroits. En particulier, les sables médians montrent des chenaux très bien marqués à deux endroits (dépression subsidente en aval de la salle Vigneron (Fig. 13) et long tunnel sous plancher). Ces endroits se situent dans une portion beaucoup plus étroite de la galerie où le courant était plus rapide (astérisques sur la Figure 4).

\section{La formation stalagmitique}

\subsection{Caractéristiques générales}

La formation stalagmitique supérieure est un complexe carbonaté comprenant à la fois des niveaux stratifiés, des brèches de stalactites et stalagmites cassées, de petites strates argileuses, et des gours fermés transformés en géodes. Cette formation se présente en une succession de fragments encore intacts, plus ou moins longs, à une altitude constante au mètre près. Les endroits de la galerie où cette formation n'est pas présente résultent de variations latérales de faciès comme dans la salle Vigneron où les blocs éboulés le remplacent, soit à des effondrements dus aux soutirages. Enfin, certains endroits ne montrent plus de témoin de la série stalagmitique, comme à la salle du minaret. Les témoins les plus en amont se situent non loin de l'entrée du trou du salpêtre, ceux les plus en aval à la salle du Styx.

Cette formation a fait l'objet de nombreuses datations U/ Th (Quinif 1991 ; 1996) réalisées en dehors de l'étude complète de la série sédimentaire. C'est pourquoi elles sont reprises ici afin de préciser le cadre géochronologique de cette dernière.
Figure 14. Salle des Mystérieuses et le plancher stalagmitique. Il se trouve audessus du personnage et est surmonté de stalagmites holocènes.

Figure 14. "Mystérieuses" room and the flowstone, located above the person It is covered by Holocene stalagmites.

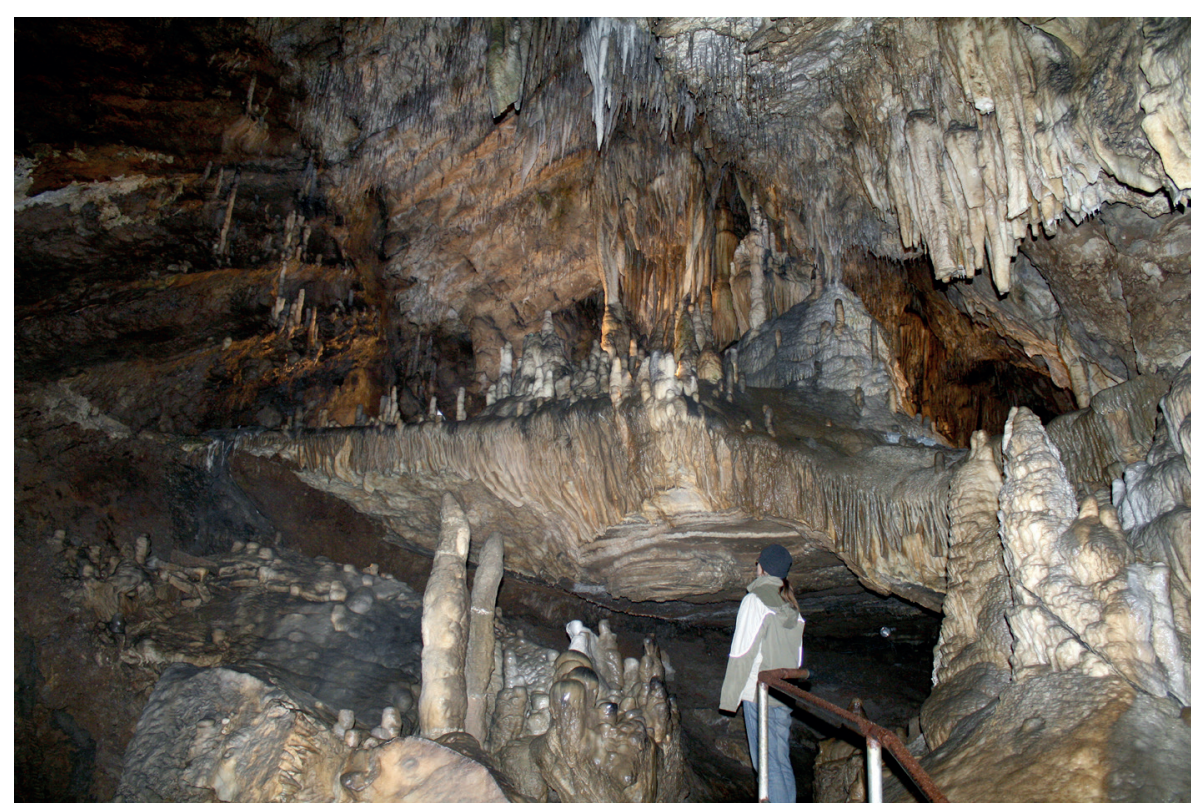




\section{H - St - 34}

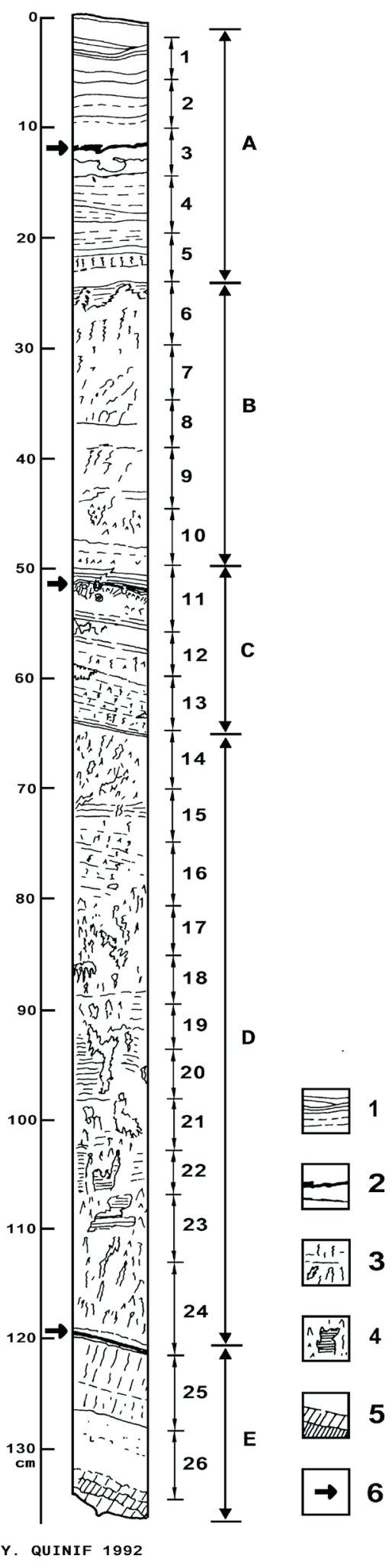

Figure 15. Lithostratigraphie de la carotte extraite du plancher de la salle des Mystérieuses. 1. Stratification nette (lignes continues) et diffuse (lignes interrompues). 2. Discontinuités. 3. Trous aux parois hérissées de cristaux. 4. Trous avec remplissage calcitique. 5. Zones colorées non soulignées par la stratification. 6. Principales discontinuités. Les numéros indiqués le long de la colonne stratigraphiques renvoient aux échantillons.

Figure 15. Lithostratigraphy of the core from the flowstone in the "Mystérieuses" room. 1. Clear stratification (continuous line) and diffuse stratification (interrupted line). 2. Discontinuities. 3. Holes with walls covered by crystals. 4 . Holes with calcite filling. 5. Coloured zones without stratification. 6. Main discontinuities. The numbers along the stratigraphic column refer to the samples.

\subsection{Lithostratigraphie et chronologie du plancher - Carottage H-St-34}

Une formation telle que celle-ci constitue un jalon important dans l'évolution de la cavité. Elle a été datée par la méthode $\mathrm{U} / \mathrm{Th}$ et a fourni ainsi une chronologie à la série sédimentaire (Quinif, 1991 ; 1996). Dans le détail, les variations sont multiples puisque la précipitation de la calcite se fait dans des conditions géométriques très différentes suivant le cadre morphologique de la galerie. Sauf là où une continuité sédimentaire latérale peut être suivie, seules les datations peuvent donner une indication sur l'appartenance à tel ou tel ensemble géochronologique. Aussi, nous nous baserons d'abord sur le plancher de la salle des Mystérieuses où se situe le plus long fragment d'un seul tenant au sud-est de la salle, avec une filiation entre les sédiments détritiques et chimiques. De plus, la présence de l'empreinte de fentes de dessiccation donne une certaine assurance pour confirmer à cet endroit la continuité de la série stalagmitique avec la formation détritique inférieure dans cette portion du remplissage.

La série chronologique la plus complète est constituée par un des carottages effectué dans la salle des Mystérieuses : H-St-34 (Figs 14 \& 15; Quinif, 1991). Vingt-six échantillons ont été prélevés sur cette carotte, dont 23 ont fait l'objet d'une analyse isotopique. De ces résultats, l'échantillon 15 doit être écarté car il constitue un artefact. La qualité de la calcite permet de penser que le plancher n'a pas été à cet endroit ouvert géochimiquement comme nous avons pu le constater à d'autres endroits.

Sur base de caractéristiques lithologiques, la série stalagmitique, épaisse de $137 \mathrm{~cm}$, a été divisée en cinq ensembles, numérotés de A (plus jeune) à E (plus vieux). Ces données résultent d'observations macroscopiques.

Ensemble $A$ de 0 à $25 \mathrm{~cm}$.

Le sommet de la série est constitué de calcite compacte, finement stratifiée, sans que l'on puisse y trouver une lamination annuelle. Ce premier ensemble est coupé à $12 \mathrm{~cm}$ par un arrêt de croissance, souligné par une couche argileuse séparant deux surfaces de discontinuité.

Ensemble B de 25 à $50 \mathrm{~cm}$.

Le faciès de la calcite est de type columnaire avec de nombreux trous aux parois hérissées de cristaux. La stratification est souvent quasiment invisible ou inexistante. Ce faciès est la marque de la croissance d'une succession stratigraphique de gours. Les trous sont la trace des gours qui se ferment.

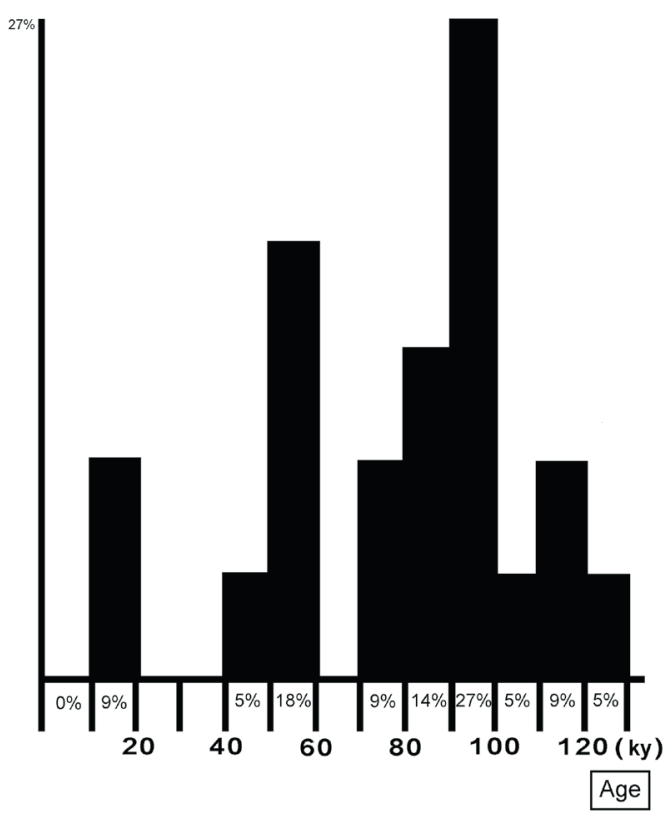

Figure 16. Distribution des âges du carottage H-St-34. Figure 16. Distribution of the core H-St-34 ages. 
Ensemble $C$ de 50 à $65 \mathrm{~cm}$.

Il débute par une strate colorée en brun pâle, formée ellemême d'une suite de petites strates dont l'une d'entre elles est divisée en lamines. La calcite est massive, translucide. Sous cette strate colorée, une stalactite fistuleuse est visible en coupe : on distingue le canal central. Le reste de cet ensemble est stratifié, la calcite est blanche, soit transparente, soit translucide. Cette stratification résulte d'une suite de fines strates inframillimétriques transparentes faites de calcite massive, sans cristaux visibles à l'œil nu, et de strates de quelques millimètres d'épaisseur, constituées de calcite blanche et translucide à opaque, palissadique, aux cristaux visibles. La cohésion mécanique est plus faible que celle de l'ensemble A. La carotte se casse facilement suivant les joints de stratification.

\section{Ensemble D de 65 à $120 \mathrm{~cm}$.}

Cet ensemble présente un faciès de gours, comme l'ensemble B. C'est une suite monotone de plages à trous séparées, à la fois verticalement et horizontalement, par des surfaces stratifiées. Cette stratification présente les mêmes caractéristiques que celle de la plage $\mathrm{C}$. Cet ensemble résulte donc de la croissance d'une succession de gours et, lorsque la surface du plancher est horizontale, d'un faciès stratifié.

\section{Ensemble E de 120 à $137 \mathrm{~cm}$.}

Il est séparé de l'ensemble précédent par une couche épaisse de $5 \mathrm{~mm}$ constituée de fines strates de calcite blanche, transparente, compacte, et de strates chargées d'argile, qui confèrent à cette suite une couleur brune. Le reste de cet ensemble est séparé en deux parties. La partie supérieure a une stratification diffuse masquée par un faciès de calcite columnaire à gros cristaux. La partie inférieure, séparée de la précédente par une strate millimétrique jaune, est constituée de calcite compacte, sans stratification, à petits trous millimétriques distribués de façon homogène. Le bas est barré par une bande de couleur brunâtre

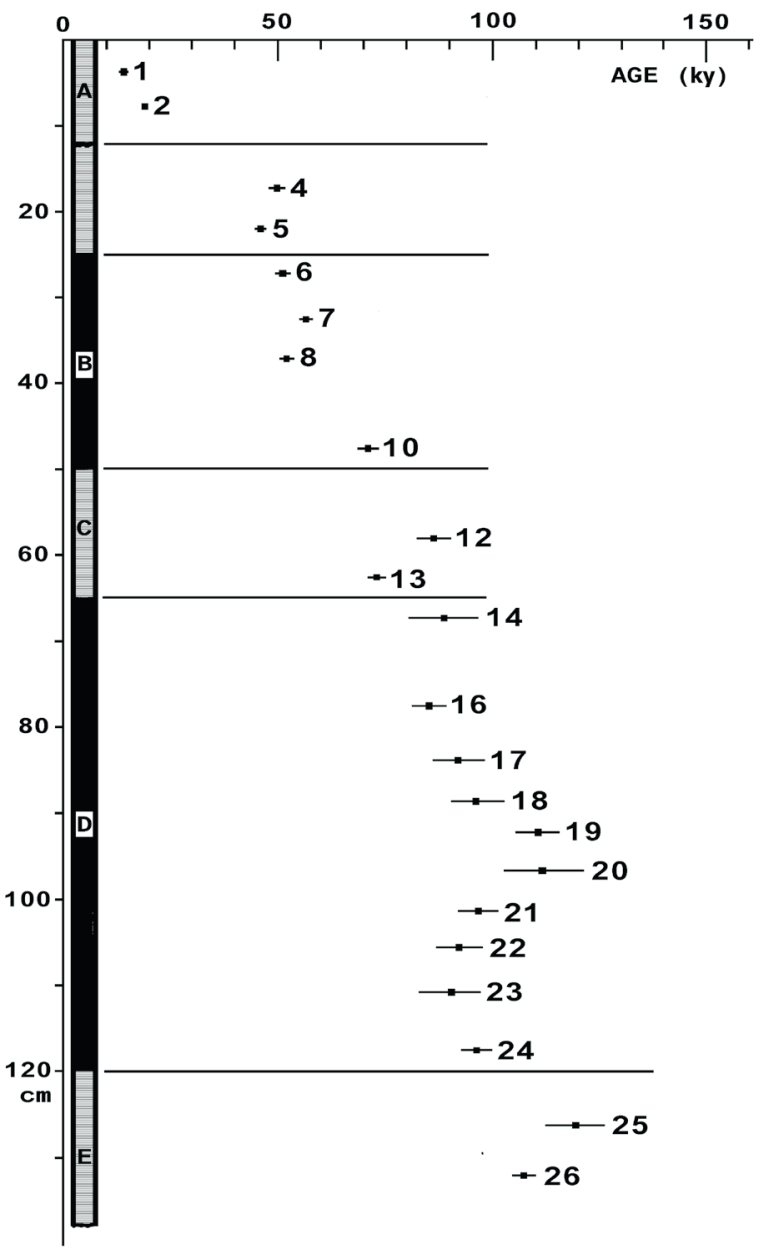

Figure 17. Chronostratigraphie du carottage H-St-34.

Figure 17. Chronostratigraphy of the core H-St-34. qui résulte d'un front d'altération. Cette partie inférieure semble résulter de deux phases de cristallisation, la seconde étant une cimentation secondaire d'une première croissance stalagmitique.

La distribution des âges indique trois pics principaux (Tableau 1; Figs 16 \& 17) : entre 10000 et 20000 ans, entre 40000 et 60000 ans et entre 70000 et 130000 ans. Les deux derniers se rapportent clairement aux stades isotopiques marins (SIM) 3 et 5 qui voient la croissance la plus importante du plancher. Trois phases de croissance sont nettement visibles, chacune correspondant aux trois pics de la distribution (Martinson et al., 1987).

Les échantillons 1 et 2 positionnent l'ensemble A dans le stade isotopique 2. Si l'âge de l'échantillon 1 n'est pas fiable vu le faible rapport isotopique ${ }^{230} \mathrm{Th} /{ }^{232} \mathrm{Th}$ indiquant une contamination détritique, on ne peut suspecter celui de l'échantillon 2. Le faciès de la calcite ne porte pas non plus à imaginer une ouverture géochimique du système. L'hypothèse la plus plausible est de rattacher le sommet du plancher à un interstade de la dernière glaciation (Dansgaard et al., 1982 ; Johnsen et al., 1992 ; Rasmussen et al., 2014). L'âge de 19200 \pm 600 ans est forcément une moyenne sur les différents niveaux investigués sur les $5 \mathrm{~cm}$ d'épaisseur du prélèvement. La lacune séparant les ensembles $\mathrm{A}$ et $\mathrm{B}$ pourrait correspondre à un arrêt de croissance suite à un événement sismique visualisé par la surface de discontinuité.

Les échantillons 4 à 8 se regroupent très fortement entre 46000 et 57000 ans [valeur moyenne $=51560 ; \sigma=4,087$ ]. C'est le sous-stade isotopique 3.3, où les récentes analyses y voient plusieurs interstades (Dansgaard et al., 1982). Il est surprenant que le plancher stalagmitique n'enregistre pas le reste du stade 3, alors que nous l'avons découvert dans d'autres spéléothèmes dans le même réseau (Quinif, 1991). Plus surprenant est l'absence de rupture lithologique entre les échantillons 8 et 10 . La surface principale la plus proche est celle qui sépare les ensembles $\mathrm{B}$ et $\mathrm{C}$ et ne se retrouve pas dans la chronologie. Or, rien ne permet de mettre en doute la datation de l'échantillon 10.

Les échantillons 10 à 26 forment le troisième groupe chronologique. La valeur moyenne est de 95527 avec un écarttype de 13628 . Il existe des inversions chronologiques ce qui rend les validations difficiles. Du point de vue lithologique, le passage de l'ensemble C à l'ensemble $\mathrm{D}$ ne constitue pas une rupture et ne peut pas être interprété en termes de modification climatique. Il s'agit plutôt d'une modification géométrique de la surface du spéléothème qui influe sur le type de fabrique cristalline. Par contre, la transition entre les ensembles $\mathrm{D}$ et $\mathrm{E}$ se marque par une rupture sédimentologique et pourrait avoir une signification climatique. Il faut écarter de l'ensemble E l'échantillon 26 qui, montrant des traces d'altération, est plus sujet à caution que le précédent. Dans ce cas, l'ensemble E marquerait le sous-stade isotopique 5.5 (5e) et les ensembles $\mathrm{D}$ et $\mathrm{C}$ le sous-stade 5.3 : âge moyen de 94764 ans avec un écart-type de 10 867. Il nous faut remarquer que l'échantillon 13, avec un âge de 74200 ans, est proche de la transition entre les stades 5 et 4 .

Ces trois familles d'âges correspondent donc respectivement à un interstade du stade 2 , au stade 3.3 et au stade 5 . Les âges des échantillons $4,5,6,7$ et 8 sont très proches et expriment une croissance très rapide du plancher. La même remarque peut être faite pour les échantillons 16, 17, 18, 21, 22, 23 et 24. Leur moyenne est de 92686 ans avec un écart-type de 4000 ans. Cet âge est donné comme le maximum du sous-stade isotopique 5.31 (96 $380 \pm 5020$ ans sur la courbe SPECMAP, Martinson et al., 1987). L'ensemble E correspond au sousstade isotopique 5.5 en ne tenant compte que de l'âge de 25 , celui de 26 étant suspect à cause des traces d'altération. Une régression linéaire de la totalité des échantillons donne un âge de 128000 ans pour la base du plancher. En ne tenant compte que de la partie inférieure, on trouve 132000 ans. Malgré la qualité moyenne des résultats, la base de ce plancher se situe au début du stade isotopique 5 . 


\section{H-St-30}

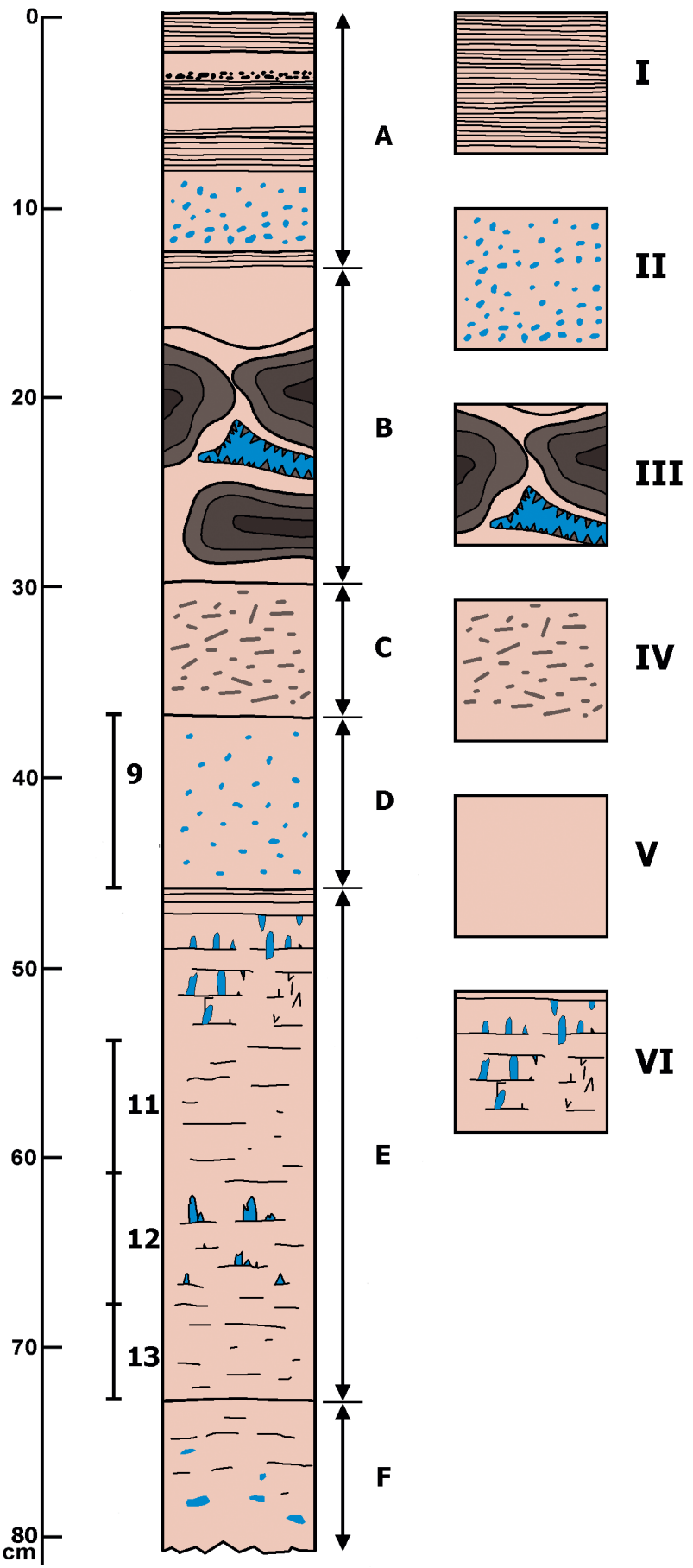

Figure 18. Lithostratigraphie du carottage H-St-30. Il a été pratiqué dans la zone de la "forêt ». Les zones en bleu symbolisent les trous. Les lignes subhorizontales se rapportent aux zones les plus stratifiées. I. Section stratifiée ; II. Calcite à petits trous ; III. Stalagmites cassées ; IV. Brèche à stalactites fistuleuses ; V. Calcite massive ; VI. Calcite à géodes. La section $\mathrm{B}$ est une zone à stalagmites cassées, tombées et cimentées par la croissance du plancher stalagmitique. La zone $\mathrm{C}$ est une brèche à stalactites fistuleuses. Ces zones sont des témoignages d'évènements sismo-tectoniques (Quinif, 1991). Les échantillons datés sont les échantillons 9, 11, 12 et 13. La section F renferme des nodules de type ferricrête.

Figure 18. Lithostratigraphy of the core H-St-30, coming from the area called "the forest". Holes are represented by blue areas. The subhorizontal lines mention the most stratified areas. I. Stratified section; II. Calcite with small holes; III. Broken stalagmites; IV Breccia broken soda-straws; V. Massive calcite; VI. Calcite with geodes. Section B is an area with broken stalagmites which have fallen down and cemented by the growth of the flowstone. Area $\mathrm{C}$ is a soda-straw breccia. Those areas are the witness of sismo-tectonics events. Dated samples are 9, 11, 12 and 13. Section F contains ferricrust nodules.

\subsection{Autres données chronologiques}

De multiples analyses chronologiques et palynologiques ont été réalisées sur ce plancher, à différents endroits de la galerie (Quinif \& Bastin, 1994). Un carottage à l'extrémité du long segment de plancher avant les Mystérieuses donnent deux âges pour la base de $124600[+8,6 ;-8,2]$ et $127900[+10,2$; $-9,4]$ avec des rapports isotopiques ${ }^{230} \mathrm{Th} /{ }^{232} \mathrm{Th}$ de 99 et 64 . Enfin, trois séries non publiées donnent pour la base un âge plus vieux que 130000 ans. En particulier, la série du carottage H-St-30 donne quatre dates (Fig. 18, Tableau 2) qui situent une croissance stalagmitique lors du stade 7 et du stade 9 . Les strates basales de ce carottage englobent des nodules soit argileux mous, soit ferrifères durs, de type ferricrête.

\section{Interprétation}

\subsection{La série détritique}

La formation détritique témoigne d'une séquence d'assèchement complexe. Les deux sous-formations sont granodécroissantes, avec des contextes d'écoulements faibles avec chenaux. C'est l'image d'un écoulement assez faible puisque, lors de ces épisodes, la rivière ne remplit pas complètement toute la section de la galerie. Les sédiments plus fins marquent des conditions d'écoulement très faible, de suspension uniforme. Cette caractéristique marque une phase d'assèchement progressif de la galerie. En comparaison avec d'autres sites analysés par la palynologie (Genty et al., 1992 ; Quinif et al., 1979 ; Quinif \& Bastin, 1989) ainsi que la série plus récente de la galerie des petites fontaines dans la même grotte (Blockmans et al., 1999), la sédimentation détritique se déroule durant une période de dégradation climatique comme une période glaciaire. La charge détritique est importante vu la prédominance de l'érosion mécanique en surface (rhexistasie, Erhart, 1967). La fin de cette période voit la diminution des écoulements, soit parce que le climat devient plus sec avant la période interglaciaire suivante, soit parce que les écoulements empruntent des passages de plus basse altitude. En l'absence de marqueurs climatiques inclus dans la formation détritique, il n'est pas possible de trancher.

\subsection{La série stalagmitique}

Elle a enregistré le stade isotopique 5 pour sa plus grande partie. Au dessus, on retrouve une croissance au stade 3. Les âges mesurés sur le plancher proche de la salle des Mystérieuses (H-St-18, 19, 20 et 21, Quinif, 1991) indiquent un début de croissance au stade 5.5 ou 5.3. Les empreintes de fentes de dessiccation (« mud-cracks ») indiquent une continuité temporelle entre la fin de la sédimentation détritique et le début de la croissance des spéléothèmes. Sans doute existe$\mathrm{t}$-il des lacunes par endroits mais le passage stade 6 - stade 5 semble probable.

Par contre, les âges de la base de certains carottages se situant dans le stade 7 et même le stade 9 indiquent que la galerie a connu d'autres séquences sédimentaires plus anciennes, par exemple la croissance d'une formation stalagmitique au stade 7 sur une formation détritique du stade 8, avec l'érosion des formations détritiques anciennes. Nous retrouvons là le télescopage de séquences climatiques dans la même galerie, ainsi que cela a déjà été prouvé dans la grotte de la Vilaine Source (Quinif et al., 1979). L'ouverture géochimique des spéléothèmes les plus anciens peut se comprendre par des immersions dues aux péjorations climatiques qui ont suivi leur formation.

\section{Conclusion}

La série sédimentaire de la galerie des Verviétois témoigne de points importants concernant l'évolution post génétique d'un système karstique de type recoupement souterrain de méandre en relief appalachien. Tout d'abord, on y décrypte une phase d'assèchement liée probablement à un important changement climatique : passage d'une période glaciaire à une période interglaciaire. Si des preuves par 


\begin{tabular}{|c|c|c|c|c|c|c|c|}
\hline Samples & height $(\mathrm{cm})$ & {$[\mathrm{U}]_{\mathrm{ppm}}$} & ${ }^{234} \mathrm{U} /{ }^{238} \mathrm{U}$ & ${ }^{230} \mathrm{Th} /{ }^{234} \mathrm{U}$ & ${ }^{230} \mathrm{Th} /{ }^{232} \mathrm{Th}$ & {$\left[{ }^{234} U /{ }^{238} U\right]_{t=0}$} & Age ky \\
\hline H-St-34(01) & 40 & $0.288 \pm 0.003$ & $2.186 \pm 0.023$ & $0.124 \pm 0.006$ & $14 \pm 3$ & 2.336 & $14.1[ \pm 0.7]$ \\
\hline H-St-34(02) & 71 & $0.327 \pm 0.003$ & $2.276 \pm 0.020$ & $0.165 \pm 0.004$ & $61 \pm 14$ & 2.346 & $19.2[+0.5 /-0.6]$ \\
\hline H-St-34(04) & 121 & $0.307 \pm 0.004$ & $2.176 \pm 0.025$ & $0.385 \pm 0.014$ & $63 \pm 16$ & 2.355 & $50.3[ \pm 2.2]$ \\
\hline H-St-34(05) & 219 & $0.369 \pm 0.004$ & $2.083 \pm 0.021$ & $0.358 \pm 0.010$ & $61 \pm 13$ & 2.233 & $46.2[ \pm 1.6]$ \\
\hline H-St-34(06) & 269 & $0.241 \pm 0.003$ & $1.976 \pm 0.020$ & $0.389 \pm 0.011$ & $88 \pm 28$ & 2.127 & $51.2[+1.7 /-1.8]$ \\
\hline H-St-34(07) & 323 & $0.230 \pm 0.003$ & $2.015 \pm 0.023$ & $0.428 \pm 0.009$ & $57 \pm 7$ & 2.192 & $57.5[+1.6 /-1.5]$ \\
\hline H-St-34(08) & 370 & $0.261 \pm 0.004$ & $1.947 \pm 0.026$ & $0.398 \pm 0.011$ & $71 \pm 13$ & 2.097 & $52.6[+1.8 /-1.7]$ \\
\hline H-St-34(10) & 471 & $0.228 \pm 0.003$ & $1.919 \pm 0.023$ & $0.507 \pm 0.011$ & $78 \pm 13$ & 2.125 & $71.9[+2.3 /-2.1]$ \\
\hline H-St-34(12) & 579 & $0.143 \pm 0.002$ & $1.685 \pm 0.030$ & $0.577 \pm 0.018$ & $39 \pm 7$ & 1.875 & $87.0[+4.0 /-3.9]$ \\
\hline H-St-34(13) & 623 & $0.165 \pm 0.002$ & $1.672 \pm 0.023$ & $0.515 \pm 0.010$ & $36 \pm 4$ & 1.828 & $74.2[+2.2 /-2.4]$ \\
\hline H-St-34(14) & 674 & $0.092 \pm 0.002$ & $1.638 \pm 0.040$ & $0.586 \pm 0.035$ & $21 \pm 7$ & 1.819 & $89.2[+8.3 /-7.7]$ \\
\hline H-St-34(15) & 721 & $0.087 \pm 0.001$ & $1.649 \pm 0.029$ & $0.949 \pm 0.038$ & $82 \pm 40$ & 2.193 & $217.1[+27.1 /-22.0]$ \\
\hline H-St-34(16) & 775 & $0.111 \pm 0.002$ & $1.633 \pm 0.032$ & $0.575 \pm 0.018$ & $35 \pm 6$ & 1.808 & $86.7[+4.2 /-4.0]$ \\
\hline H-St-34(17) & 825 & $0.109 \pm 0.002$ & $1.574 \pm 0.027$ & $0.602 \pm 0.025$ & $20 \pm 4$ & 1.745 & $93.1[+6.2 /-5.8]$ \\
\hline H-St-34(18) & 869 & $0.109 \pm 0.002$ & $1.596 \pm 0.032$ & $0.621 \pm 0.024$ & $29 \pm 6$ & 1.784 & $97.6[+6.0 /-5.7]$ \\
\hline H-St-34(19) & 916 & $0.102 \pm 0.002$ & $1.614 \pm 0.028$ & $0.678 \pm 0.018$ & $36 \pm 5$ & 1.840 & $111.6[+5.1 /-4.8]$ \\
\hline H-St-34(20) & 960 & $0.106 \pm 0.002$ & $1.578 \pm 0.041$ & $0.679 \pm 0.032$ & $64 \pm 34$ & 1.792 & $112.4[+9.4 /-8.7]$ \\
\hline H-St-34(21) & 1003 & $0.095 \pm 0.002$ & $1.683 \pm 0.038$ & $0.624 \pm 0.018$ & $43 \pm 7$ & 1.898 & $97.6[+4.7 /-4.4]$ \\
\hline H-St-34(22) & 1044 & $0.093 \pm 0.002$ & $1.650 \pm 0.033$ & $0.604 \pm 0.022$ & $24 \pm 4$ & 1.845 & $93.2[+5.3 /-5.0]$ \\
\hline H-St-34(23) & 1091 & $0.104 \pm 0.002$ & $1.618 \pm 0.027$ & $0.596 \pm 0.031$ & $42 \pm 14$ & 1.800 & $91.6[+7.4 /-6.9]$ \\
\hline H-St-34(24) & 1172 & $0.091 \pm 0.002$ & $1.620 \pm 0.034$ & $0.621 \pm 0.014$ & $47 \pm 5$ & 1.815 & $97.4[+3.6 /-3.5]$ \\
\hline H-St-34(25) & 1239 & $0.094 \pm 0.002$ & $1.613 \pm 0.034$ & $0.712 \pm 0.023$ & $84 \pm 22$ & 1.860 & $121.0[+7.1 /-6.7]$ \\
\hline H-St-34(26) & 1313 & $0.166 \pm 0.002$ & $1.650 \pm 0.019$ & $0.667 \pm 0.010$ & $81 \pm 8$ & 1.881 & $108.4[+2.8 /-2.7]$ \\
\hline
\end{tabular}

Tableau 1. Données isotopiques de l'analyse du carottage H-St-34. La première colonne comprend le nom de l'échantillon, la deuxième colonne donne la teneur en uranium exprimée en ppm, les troisième, quatrième et cinquième colonnes donnent les différents rapports isotopiques mesurés, la sixième le rapport isotopique entre l'uranium-234 et l'uranium-238 à l'instant initial et, enfin, la septième, l'âge de l'échantillon. Les erreurs sont indiquées à $1 \sigma$.

Table 1. Isotopic results and ages of the speleothems. The first column gives the name of the sample, the second the uranium content in ppm, the third, fourth and fifth are the different measured isotopic ratios, the sixth the initial isotopic ratio between ${ }^{234} \mathrm{U} /{ }^{238} \mathrm{U}$ and the seventh the age. Errors are indicated at $1 \sigma$.

exemple palynologiques n'ont pas pu être apportées ici, il y a une forte convergence d'arguments issus d'autres séquences souterraines pour que cette interprétation soit correcte (Bastin et al., 1988 ; Blockmans et al., 1999 ; Genty et al., 1992 ; Bastin et al., 1977 ; Quinif, 1990 ; 1994 ; 2006 ; Quinif et al., 1979 ; Quinif \& Bastin, 1989 ; 1993). Bien que les marqueurs climatiques directs comme l'analyse pollinique manquent ici, la comparaison avec d'autres coupes souterraines de Belgique y compris dans la grotte de Han parle dans ce sens. Elle illustre une fois de plus la capacité d'enregistrement climatique des remplissages souterrains jouissant des possibilités de datation spécifiques du milieu endokarstique. La galerie des Verviétois, de niveau supérieur de 10 mètres à la surface piézométrique d'étiage actuelle, a enregistré des phénomènes sédimentaires appartenant aux stades isotopiques 9, 7, 5, 3 et 2. La grotte de Bohon, grotte de recoupement souterrain de méandre de l'Ourthe, présente également une série sédimentaire située quelques mètres au-dessus du niveau actif et appartenant aux stades isotopiques 6, 5, 4, 3, 2, 1 (Bastin et al., 1988). La grotte de la Vilaine Source, axée sur la résurgence du ruisseau souterrain du vallon de Lesves, a livré une autre série sédimentaire appartenant également aux stades de 6 à l'actuel (Quinif et al., 1979). Ces constatations contribuent à argumenter la thèse de l'enfoncement irrégulier de l'enfoncement des rivières en Haute Belgique. En effet, dans une même tranche altitudinale, les remplissages prouvent l'existence de plusieurs phases climatiques. La succession régulière des terrasses fluviatiles suivant celle des périodes glaciaires s'en trouve remise en question. La grotte du Nou Maulin et de Lorette à Rochefort constituent également un cas analogue significatif. La perte du Nou Maulin absorbe lors des crues l'eau de la Lomme. En étiage, une digue canalisant la rivière aérienne, la surface piézométrique se situe à $10 \mathrm{~m}$ sous le talweg extérieur. Dans cette grotte, plusieurs spéléothèmes situés quelques $6 \mathrm{~m}$ au-dessus du talweg extérieur de la Lomme ont été datés de plus de 400000 ans (Quinif, 2016). De ce fait, elle prouve le caractère irrégulier de l'enfoncement des rivières en Haute Belgique puisque plusieurs phases climatiques se retrouvent enregistrées à la même altitude. Un cas analogue a été mis en évidence dans la grotte de la Vilaine source où une série sédimentaire complexe a enregistré à la même altitude les phases isotopiques de 7 à 1 (Quinif et al., 1979 ; 1987 ; Quinif, 2013), comme dans la grotte de Lorette à Rochefort (Quinif et al., 2011). Enfin, c'est un exemple supplémentaire qui apporte des éléments pour comprendre le fonctionnement sédimentaire de l'endokarst durant le Pléistocène dans un environnement géographique et climatique analogue à nos karsts de bas plateau à une latitude analogue.

\begin{tabular}{cccccc}
\hline Samples & {$[\mathrm{U}]_{\mathrm{ppm}}$} & ${ }^{234} \mathrm{U} /{ }^{238} \mathrm{U}$ & ${ }^{230} \mathrm{Th} /{ }^{234} \mathrm{U}$ & ${ }^{230} \mathrm{Th} /{ }^{232} \mathrm{Th}$ & ${ }^{234} \mathrm{U} /{ }^{238} \mathrm{U}_{\mathrm{t}=0}$ \\
\hline H-St-30(9) & $0.070( \pm 0.001)$ & $1.541( \pm 0.032)$ & $0.858( \pm 0.022)$ & $37.0( \pm 4.7)$ & 1.883 \\
H-St-30(11) & $0.215( \pm 0.004)$ & $1.797( \pm 0.034)$ & $1.009( \pm 0.027)$ & $13.7( \pm 0.8)$ & 2.604 \\
H-St-30(12) & $0.232( \pm 0.003)$ & $1.837( \pm 0.023)$ & $0.977( \pm 0.069)$ & $25.7( \pm 9.2)$ & 2.576 \\
H-St-30(13) & $0.255( \pm 0.005)$ & $1.932( \pm 0.043)$ & $1.076( \pm 0.086)$ & $38.9( \pm 21)$ & 3.156 \\
\hline
\end{tabular}

Tableau 2. Données isotopiques du carottage H-St-30. Même légende qu'au Tableau 1.

Table 2. Isotopic data of the core H-St-30. Same legend as Table 1. 


\section{Remerciements}

Ces travaux n'auraient pu voir le jour sans l'autorisation de la direction et l'aide du personnel de la SA Domaine des Grottes de Han. Qu'ils soient remerciés pour leur collaboration et leur accueil toujours aimable et plein d'empathie sur le site. Les travaux souterrains ont été réalisés avec l'aide des spéléologues de l'Equipe spéléo du Centre et de Mons (ESCM), de collaborateurs du Service de Géologie fondamentale et appliquée de la Faculté Polytechnique de Mons, de scientifiques venus d'autres universités et instituts de recherches, de spéléos issus de divers horizons et de techniciens de la Société des grottes de Han. Qu'ils soient tous certains de ma reconnaissance. Enfin, mes remerciements vont également aux deux reviewers qui ont su corriger maints défauts dans mon texte.

\section{Bibliographie}

Bastin, B., Quinif, Y., Dupuis, C. \& Gascoyne, M., 1988. La séquence sédimentaire de la Grotte de Bohon (Belgique). Annales de la Société Géologique de Belgique, 111/1, 51-60.

Bastin, B., Dupuis, C. \& Quinif ,Y., 1977. Application des méthodes de la géologie du Quaternaire à l'étude de la spéléogenèse exemples pris dans les grottes belges. Congrès International de l'Union Internationale de Spéléologie, Sheffield, 24-28.

Blockmans, S. \& Dumoulin, V., sous presse. Carte géologique de Wallonie : Houyet - Han-sur-Lesse 59/1-2. 1/25.000. Namur, Service Public de Wallonie, Direction générale opérationnelle Agriculture, Ressources naturelles et Environnement, avec une notice explicative.

Blockmans, S., Quinif, Y., Bini, A. \& Zuccoli, L., 1999. Dynamique sédimentaire et paléoenvironnements durant la transition Weichsélien - Holocène à partir des dépôts endokarstiques de la grotte de Han-sur-Lesse (Belgique). Bulletin de la Société Géologique de France, 170/6, 841-852.

Bonniver, I., 2011. Etude hydrogéologique et dimensionnement par modélisation du « système-traçage » du réseau karstique de Hansur-Lesse (Massif de Boine - Belgique). Université de Namur, Thèse de doctorat en Sciences, Presses universitaires de Namur, $349 \mathrm{p}$.

Boulvain, F. \& Pingot J.-L., 2011. Genèse du sous-sol de la Wallonie. Académie royale de Belgique, Mémoires de la Classe des sciences in $8^{\circ}, 34,190 \mathrm{p}$.

Boulvain, F., Mabille, C., Poulain, G. \& Da Silva, A.-C., 2009. Towards a palaeogeographical and sequential framework for the Givetian of Belgium. Geologica Belgica, 12/3-4, 161-178.

Dandurand, G., Devès, G., Maire, R., Ortega, R., Genty, D. \& Ghaleb, B., 2011. Étude morphosédimentaire d'un remplissage endokarstique de la grotte du Bois du Clos (Charente, France) : contraintes géométriques et dynamiques hydrologiques. Quaternaire, 22/4, 285-306.

Dansgaard, W., Clausen, H.B., Gundestrup, N., Hammer, C.U., Johnsen, S.J., Kristinsdottir, P.M. \& Reeh, N., 1982. A new Greenland deep ice core. Science, 218, 1273-1277.

Delvaux de Fenffe, D., 1985. Géologie et tectonique du parc de Lesse et Lomme au bord sud du Bassin de Dinant (Rochefort, Belgique). Bulletin de la Société belge de Géologie, 94/1, 81-95.

Delvaux de Fenffe, D., 1989. Structures tardi- et post- hercyniennes dans le bord sud du synclinorium de Dinant, entre Han-sur-Lesse et Beauraing (Belgique). Annales de la Société Géologique de Belgique, 112/2, 317-325.

Delvaux de Fenffe, D., 1998. Structure et évolution tectonique de la région de Lesse-et-Lomme. Speleochronos, hors-série 1998, 49-51.

Derruau, M., 1974. Précis de géomorphologie, 6 e éd. Masson, Paris, $453 \mathrm{p}$.

Ek, C. \& Quinif, Y., 1988. Les sédiments détritiques de grottes : aperçu synthétique. Annales de la Société Géologique de Belgique, 111/1, 1-7.

Erhart, H., 1967. La genèse des sols en tant que phénomène géologique. Masson, Paris, Evolution des sciences, 90 p.

Genty, D., Quinif, Y. \& Bastin, B., 1992. Un remplissage endokarstique tardiglaciaire et holocène (nouvelle galerie de la Grotte de Rochefort). Speleochronos, 4, 31-40.

Imbrie, J., Hays, J.D., Martinson, D.G., McIntyre, A., Mix, A.C., Morley, J.J., Pisias, N.G., Prell, W.L. \& Shackleton N.J., 1984 The orbital theory of Pleistocene climate: Support from a revised chronology of the marine $\delta^{18} \mathrm{O}$ record. In Berger, A.L., Imbrie, J., Hays, J., Kukla, G., Saltzman, B. (eds), Milankovitch and Climate, Part 1. Reidel, Dordrecht, NATO ASI series, 126, 269-305.

Johnsen, S.J., Clausen, H.B., Dansgaard, W., Iversen, P., Jouzel, J., Stauffer, B. \& Steffensen, J.P., 1992. Irregular glacial interstadial recorded in a new Greenland ice core. Nature, 359, 311-313.
Lannoote, E., Kaufmann, O., Quinif, Y., 2013. Contexte et fonctionnement hydrogéologique d'un système karstique perterésurgence (Wellin, Belgique). Karstologia, 61, 25-36.

Lauritzen, S.-E. (ed.), 1996. Climate Change: The Karst Record Karst Waters Institute Special Publication, 2, 203 p.

Losson, B., Corbonnois, J., Argant, J., Brulhet, J., Pons-Branchu, E. \& Quinif Y., 2006. Interprétation paléoclimatique des remplissages endokarstiques de la vallée de la Moselle à Pierrela-Treiche (Lorraine, France). Géomorphologie : relief, processus, environnement, 1/2006, 37-48.

Maire, R. \& Pomel, S., 1997. Exemple d'enregistrement des changements climatiques et de l'anthropisation dans les remplissages endokarstiques de Chine centrale (Hubei). Quaternaire, 8/2-3, 119-128.

Martinson, D.G., Pisias, N.G., Hays, J.D., Imbrie, J., Moore, T.C. \& Shackelton, N.J., 1987. Age dating and the orbital theory of the ice ages: development of a high resolution 0 to 300,000-years chronostratigraphy. Quaternary Research, 27, 1-29.

Passega, R., 1957. Texture as characteristic of clastic deposition. Bulletin, American Association of Petroleum Geologists, 41/9, 1952-1984.

Passega, R., 1964. Grain size representation by CM patterns as a geological tool. Journal of Sedimentary Petrology, 34/4, 830-847.

Passega, R, 1977. Significance of CM diagrams of sediments deposited by suspension. Sedimentology, 24, 723-733.

Passega, R. \& Byramjee, R., 1969. Grain-size image of clastic deposits Sedimentology, 13, 233-252.

Quinif, Y., 1988. Une nouvelle topographie de la Grotte de Han. Lapiaz, hors-série "Spécial Han", 15-18.

Quinif, Y., 1990. La datation des spéléothèmes (U/Th) appliquée aux séquences sédimentaires souterraines pour une mise en évidence des ruptures paléoclimatiques. Karstologia Mémoires, 2, 23-32.

Quinif, Y., 1991. La série stalagmitique de la Galerie des Verviétois (Han-sur-Lesse, Belgique). Speleochronos, 3, 29-42.

Quinif, Y., 1994. Les dépôts karstiques. Concepts et méthodologies. In Maire, R., Pomel, S. \& Salomon, J.-N. (eds). Enregistreurs et Indicateurs de l'Evolution de l'Environnement en Zone Tropicale. Presses Universitaires de Bordeaux, Talence, 55-72.

Quinif, Y., 1996. La série stalagmitique de la galerie des Verviétois (Han-sur-Lesse). Speleochronos, 7, 11-20.

Quinif, Y., 2006. Complex stratigraphic sequences in Belgian caves Correlation with climatic changes during the middle, the upper Pleistocene and the Holocene. Geologica Belgica, 9/3-4, 231-244.

Quinif, Y., 2013. La grotte de la Vilaine Source. In Michel, G., Thys, G. \& CWEPSS (eds), Atlas du Karst Wallon: Bassins du Burnot et de la Molignée. Service Public de Wallonie, Namur, 61-64.

Quinif, Y., 2015. Le système karstique de Han-sur-Lesse. In Michel, G., Thys, G. \& CWEPSS (eds), Atlas du karst wallon : bassin de la Lesse Calestienne. Service Public de Wallonie, Namur, 31-38.

Quinif Y., 2016. Etagement dans la grotte de Lorette (Rochefort) Relation avec les dépôts souterrains. Regards, 81, 60-69.

Quinif, Y. \& Bastin, B., 1986. Le système karstique de Han-Sur-Lesse (Belgique). Actes 9ème Congreso Internacional de Espeleología, Barcelona, 1, 158-161

Quinif, Y. \& Bastin, B., 1989. Modalités et chronologie de la sédimentation souterraine en Belgique au Pléistocène moyen et supérieur. Acta Carsologia, 18, 71-87.

Quinif Y. \& Bastin B., 1993. Une fin d'interglaciaire : le plancher stalagmitique de Feluy - La transition entre les stades isotopiques 5 et 4 . Speleochronos, 5, 19-24.

Quinif, Y. \& Bastin B., 1994. Datation uranium/thorium et analyse pollinique d'une séquence stalagmitique du stade isotopique 5 (Galerie des Verviétois, Grotte de Han-sur-Lesse, Belgique). Comptes rendus de l'Académie des sciences, Paris, série II, 318, 211-217.

Quinif, Y. \& Maire, R., 1998. Pleistocene deposits in Pierre SaintMartin Cave, French Pyrenees. Quaternary Research, 49, 37-50

Quinif, Y. \& Vandycke, S., 2001. Les phénomènes karstiques de la région de Han-sur-Lesse - Rochefort (Belgique). Bulletin d'Information des Géologues du Bassin de Paris, 38/1, 6-19.

Quinif, Y., Dupuis, C., Bastin, B. \& Juvigné, E., 1979. Etude d'une coupe dans les sédiments quaternaires de la grotte de la Vilaine Source (Arbre, Belgique). Annales de la Société Géologique de Belgique, 102, 229-241.

Quinif, Y., Bastin, B., Dupuis, C. \& Gascoyne, M., 1987. La Grotte de la Vilaine Source. Livret-guide du Colloque International de Sédimentologie Karstique (CISK), Han-Sur-Lesse, Belgique. Centre Belge d'Etudes Karstologiques, 45-54.

Quinif, Y., Genty, D. \& Maire, R., 1995. Les spéléothèmes : un outil performant pour les études paléoclimatiques. Bulletin de la Société Géologique de France, 165/6, 603-612.

Quinif, Y., Kaufmann, O. \& Sagot, D., 2011. Les dépôts de la grotte de Lorette (Rochefort). Geological survey of Belgium professionnal paper 2011/2, 309, 55-63. 
Rasmussen, S.O., Bigler, M., Blockley, S.P., Blunier, T., Buchardt, S.L., Clausen, H.B., Cvijanovic, I., Dahl-Jensen, D., Johnsen, S.J., Fischer, H., Gkinis, V., Guillevic, M., Hoek, W.Z., Lowe, J.J., Pedro, J.B., Popp, T., Seierstad, I.K., Steffensen, J.P., Svensson, A.M., Vallelonga, P., Vinther, B.M., Walker, M.J.C., Wheatley, J.J. \& Winstrup, M., 2014. A stratigraphic framework for abrupt climatic changes during the Last Glacial period based on three synchronized Greenland ice-core records: refining and extending the INTIMATE event stratigraphy. Quaternary Science Reviews, 106, 14-28.

Rivière, A., 1977. Méthodes granulométriques. Techniques et interprétations. Masson, Paris, $167 \mathrm{p}$.

Spicer, R.A., 2003. Changing climate and biota. In: Skelton, P. (ed.), The Cretaceous World. Cambridge University Press, Cambridge, 85-163.

Van Den Broeck, E., Martel, E.A., \& Rahir, E., 1910. Les cavernes et rivières souterraines de la Belgique. Ed. Lamertin, Bruxelles, $1826 \mathrm{p}$.

Yans J. (2003). Chronologie des sédiments kaoliniques faciès wealdiens (Barrémien moyen Albien supérieur ; bassin de Mons) et de la saprolite polyphasée (Crétacé inférieur et Miocène inférieur) de la Haute-Lesse (Belgique). Implications géodynamiques et paléoclimatiques. Thèse de doctorat inédite, Faculté Polytechnique de Mons \& Université de Paris-Sud Orsay, $316 \mathrm{p}$. 